\title{
Debye Potentials
}

\section{GALVIN H. WILGOX}

\author{
Communicated by A. ERDÉLYI
}

1. Introduction. It is well known that if $u$ and $v$ are functions that satisfy the Helmholtz equation

$$
\nabla^{2} u+k^{2} u=0
$$

in a domain $D$ and if $\mathrm{r}$ is a position vector relative to an origin lying outside $D$ then the vector fields

$$
\mathbf{A}=\nabla \times(\nabla \times u \mathbf{r})+k \nabla \times v \mathbf{r}, \quad \mathbf{B}=\nabla \times(\nabla \times v \mathbf{r})+k \nabla \times u \mathbf{r},
$$

satisfy Maxwell's equations

$$
\nabla \times \mathbf{A}=k \mathbf{B}, \quad \nabla \times \mathbf{B}=k \mathbf{A},
$$

for a time-harmonic electromagnetic field in $D$. The functions $u$ and $v$ were introduced into electromagnetic theory by P. DeвYe in 1909 [10] and are called Debye potentials for the electromagnetic field (A, B). They are applied frequently in solving boundary value problems for Maxwell's equations [6, 19]. This is done, typically, by assuming that the unknown electromagnetic field has a representation by Debye potentials, using the conditions of the problem to determine what $u$ and $v$ must be, and then verifying that the fields $\mathbf{A}$ and $\mathbf{B}$ generated by this pair of potentials actually solve the original problem. This indirect procedure is designed to avoid the question of how general an electromagnetic field can be represented by Debye potentials. The present paper provides an answer to this question by showing that every electromagnetic field defined in a region between two concentric spheres can be represented there by Debye potentials. The theorem is shown to follow from a very general decomposition theorem for vector fields defined on a sphere (the Hodge decomposition theorem). Two applications of the theorem are given. They are, first, a proof that every electromagnetic field defined in a spherical domain can be expanded in a series of electromagnetic multipoles and, second, several theorems on the behavior of electromagnetic fields near isolated singular points.

The electromagnetic multipoles are the fields

$$
\mathbf{A}_{n}=\nabla \times\left(\nabla \times u_{n} \mathbf{r}\right), \quad \mathbf{B}_{n}=k \nabla \times u_{n} \mathbf{r},
$$


where

$$
u_{n}(\mathbf{r})=\frac{Z_{n+\frac{1}{2}}(k r)}{\sqrt{r}} S_{n}(\theta, \phi),
$$

$S_{n}$ is a surface spherical harmonic, and $Z_{n+\frac{1}{2}}(x)$ is a Bessel function of order $n+\frac{1}{2}$. There is a close relationship between the representation of electromagnetic fields by Debye potentials and their representation by multipole expansions. Indeed, it is known that every solution of the Helmholtz equation can be expanded in a series of the functions (1.2). Thus if (A, B) has a representation (1.1), an expansion of (A, B) in multipoles is obtained by expanding $u$ and $v$ in series of the functions (1.2) and interchanging the summation and differentiation operations (provided the interchange is justifiable). Conversely, if (A, B) has a multipole expansion, a representation (1.1) is obtained by interchanging summation and differentiation in this expansion. Hence the two representations are, in essence, equivalent.

Expansions in spherical harmonics have long been used in treating vector differential equations. One of the first such applications is due to A. CleBsCH (1863) who proved the following result [7]. Every vector field* A can be expanded into a series $\mathbf{A}=\sum_{n=0}^{\infty} \mathbf{A}_{n}$ where

$$
\mathbf{A}_{n}(\mathbf{r})=\alpha_{n}(r) \nabla u_{n}(\mathbf{r})+\beta_{n}(r) r^{2 n+1} \nabla \frac{v_{n}(\mathbf{r})}{r^{2 n-1}}+\gamma_{n}(r) \mathbf{r} \times \nabla w_{n}(\mathbf{r})
$$

Here $\alpha_{n}, \beta_{n}$, and $\gamma_{n}$ are functions of the spherical coordinate $r$ alone, while $u_{n}, v_{n}$, and $w_{n}$ are solid spherical harmonics of degree $n$. If $\mathbf{A}$ satisfies a vector differential equation then $\alpha_{n}, \beta_{n}$, and $\gamma_{n}$ are related by certain ordinary differential equations. In particular, if $\mathbf{A}$ is the electric (or magnetic) part of a timeharmonic electromagnetic field then the expansion $\mathrm{A}=\sum_{n=0}^{\infty} \mathrm{A}_{n}$ can be shown to be equivalent to a multipole expansion for A. CleBsCH applied his expansion, in the paper mentioned above, to the description of waves in an elastic solid and obtained a complete series solution for the problem of the scattering of elastic plane waves by a rigid sphere.

A method very similar to that of CleBsCH was developed by C. W. BorchardT (1873) in a paper on the thermal expansion of an elastic solid [4]. BorchardT was led to consider vector fields A satisfying

$$
\nabla \times(\nabla \times \mathbf{A})=0, \quad \nabla \cdot \mathbf{A}=\mathbf{0}
$$

and showed that every such field could be written

$$
\mathbf{A}=\nabla u+\mathbf{r} \times \nabla v
$$

where $u$ and $v$ are solutions of Laplace's equation. If $u$ and $v$ are expanded in solid harmonics, the series are substituted in (1.4), and the summations and differentiations are interchanged, an expansion for $\mathbf{A}$ results that is easily seen

* Precise conditions for the validity of the expansion are not given. 
to be the Clebsch expansion for solutions of (1.3). Conversely, the Clebsch expansion can be used to derive the representation (1.4) for solutions of (1.3).

Another method that is closely related to that of CLeBsch was devised by H. Lamb (1881). Lamb considered the simultaneous equations [15]

$$
\begin{gathered}
\nabla^{2} u+h^{2} u=0, \quad \nabla^{2} v+h^{2} v=0, \quad \nabla^{2} w+h^{2} w=0, \\
\frac{\partial u}{\partial x}+\frac{\partial v}{\partial y}+\frac{\partial w}{\partial z}=0,
\end{gathered}
$$

which are equivalent to the vector equation

$$
\nabla \times(\nabla \times \mathbf{A})-h^{2} \mathbf{A}=0
$$

for the vector field $\mathbf{A}$ having $u, v$, and $w$ as rectangular components. The last equation is equivalent to Maxwell's equations for a time-harmonic electromagnetic field, although LAMB did not obtain it in this way. BorCHARDT's equations (1.3) are a special case of $(1.5)(h=0)$ and LAMB, who at the time of the work under discussion (1881) appears to have been familiar with BoRchaRdT's work but not that of CLEBSCH, sought to generalize BoRCHARDT's representation (1.4) to a representation for solutions of (1.6). His result may be expressed as follows [15]: Every solution $\mathbf{A}$ of (1.6) may be expanded in a series $\mathbf{A}=\sum_{n=0}^{\infty}\left(\mathbf{A}_{n}+\mathbf{A}_{n}^{\prime}\right)$ where

$$
\begin{aligned}
& \mathbf{A}_{n}(\mathbf{r})=\psi_{n-1}(h r) \nabla \phi_{n}(\mathbf{r})+\psi_{n+1}(h r) r^{2 n+3} \nabla \frac{\phi_{n}(\mathbf{r})}{r^{2 n+1}}, \quad \text { and } \\
& \mathbf{A}_{n}^{\prime}(\mathbf{r})=\psi_{n}(h r) \mathbf{r} \times \nabla \chi_{n}(\mathbf{r}) .
\end{aligned}
$$

Here $\chi_{n}$ and $\phi_{n}$ are solid harmonics while $\psi_{n}(x)$ is a solution of

$$
\frac{d^{2} \psi_{n}}{d x^{2}}+\frac{2(n+1)}{x} \frac{d \psi_{n}}{d x}+\psi_{n}=0
$$

It is easy to verify that this expansion is equivalent to the Clebsch expansion for solutions of (1.6) and to the expansion of $\mathbf{A}$ in electromagnetic multipoles. LAMB was also the first to remark that the radial components

$$
\phi=\mathbf{r} \cdot \mathbf{A}, \quad \psi=\mathbf{r} \cdot(\nabla \times \mathbf{A})
$$

play a fundamental role in the theory of solutions of (1.6). This is a consequence of his discovery that $\phi$ and $\psi$ determine A uniquely, are solutions of the Helmholtz equation, and that the harmonics occurring in (1.7) are simply multiples of the harmonics occurring in the expansions of $\phi$ and $\psi$. Thus problems involving the vector equation (1.6) are reducible to a pair of scalar problems. LAMB applied his method successfully to a variety of problems in mathematical physics, including the oscillations of a viscous spheroid [15], 1881, the vibrations of an elastic sphere [16], 1882, and the motion of a viscous fluid contained in a spherical shell [17], 1884.

The first application of LAMB's method to an electromagnetic problem is due to J. J. Thomson [23], 1893, who used the method to find the wave scattered by 
a perfectly conducting sphere when it is illuminated by a plane electromagnetic wave. Thomson's solution was extended to spheres having arbitrary conductivity and dielectric constant by G. MIE [20], 1908, who applied his solution to a study of the optical properties of colloidal suspensions. MIE produced a very real simplification in the formulas occurring in sphere problems by employing as the unknown functions the components of the field $\mathbf{A}$ relative to a system of spherical coordinates $(r, \theta, \phi)$, rather than the rectangular components used by earlier writers. These spherical components are defined by

$$
\mathbf{A}=A_{1} \hat{\mathbf{r}}+A_{2} \hat{\boldsymbol{\theta}}+A_{3} \hat{\boldsymbol{\phi}},
$$

where $\hat{\mathbf{r}}, \hat{\boldsymbol{\theta}}$, and $\hat{\boldsymbol{\phi}}$ are unit vectors in the direction of increasing $r, \theta$, and $\boldsymbol{\phi}$, respectively.* Mis, like LAMB, took the radial components $\mathbf{r} \cdot \mathbf{A}=r A_{1}$ and $\hat{\mathbf{r}} \cdot \mathbf{B}=r B_{1}$ (where $k \mathbf{B}=\nabla \times \mathbf{A}$ ) as the principal unknowns of his problem. He showed that if

$$
A_{1}=\frac{z_{n}(k r)}{r} S_{n}(\theta, \phi), \quad B_{1}=0,
$$

where $S_{n}$ is a surface spherical harmonic and

$$
z_{n}(x)=\sqrt{\frac{\pi}{2 x}} Z_{n+\frac{1}{2}}(x)
$$

is a spherical Bessel function, then

$$
A_{2}=\frac{1}{n(n+1)} \frac{1}{r} \frac{d\left(r z_{n}\right)}{d r} \frac{\partial S_{n}}{\partial \theta}, \quad A_{3}=\frac{1}{n(n+1)} \frac{1}{r} \frac{d\left(r z_{n}\right)}{d r} \frac{1}{\sin \theta} \frac{\partial S_{n}}{\partial \phi}
$$

while if

$$
A_{1}^{\prime}=0, \quad B_{1}^{\prime}=\frac{z_{n}}{r} S_{n}
$$

then

$$
A_{2}^{\prime}=\frac{k z_{n}}{n(n+1)} \frac{1}{\sin \theta} \frac{\partial S_{n}}{\partial \phi}, \quad A_{3}^{\prime}=\frac{-k z_{n}}{n(n+1)} \frac{\partial S_{n}}{\partial \theta} .
$$

One can verify easily that the fields $\mathbf{A}, \mathbf{A}^{\prime}$ so defined are equivalent to LAMB's solutions (1.7). The spherical components of $\mathbf{A}$ and $\mathbf{A}^{\prime}$ are obviously simpler to describe than the corresponding rectangular components.

BorchaRDT's theorem differs from those of CleBSCH and LAMB in that it represents the general solution of his system of differential equations (1.3) in terms of a pair of scalar functions, with no explicit reference to spherical harmonics. An analogous representation for solutions of Maxwell's equations was obtained by P. Debye [10], 1909, in a paper concerning the pressure exerted by

\footnotetext{
* Throughout this paper, if $\alpha$ is a coordinate than $\hat{\boldsymbol{\alpha}}$ is a unit vector in the direction of increasing $\alpha ; \hat{\boldsymbol{\alpha}}=\nabla \alpha /(\nabla \alpha \cdot \nabla \alpha)^{1 / 2}$.
} 
electromagnetic waves on spheres of arbitrary material. DEBYe began by writing Maxwell's equations for the electric and magnetic fields $\mathbf{A}$ and $\mathbf{B}$ in terms of their spherical components. He then observed that if one makes the substitution

$$
A_{1}=\left(\frac{\partial^{2}}{\partial r^{2}}+k^{2}\right)(r u), \quad B_{1}=0
$$

where the function $u$ satisfies the Helmholtz equation, then Maxwell's equations for the remaining components of A may be integrated, yielding

$$
A_{2}=\frac{\partial}{\partial \theta} \frac{1}{r} \frac{\partial(r u)}{\partial r}, \quad A_{3}=\frac{1}{\sin \theta} \frac{\partial}{\partial \phi} \frac{1}{r} \frac{\partial(r u)}{\partial r} .
$$

Similarly if

$$
A_{1}^{\prime}=0, \quad B_{1}^{\prime}=\left(\frac{\partial^{2}}{\partial r^{2}}+k^{2}\right)(r v),
$$

where $v$ satisfies the Helmholtz equation, then

$$
A_{2}^{\prime}=\frac{k}{\sin \theta} \frac{\partial v}{\partial \phi}, \quad A_{3}^{\prime}=-k \frac{\partial v}{\partial \theta} .
$$

On adding the fields $\mathbf{A}$ and $\mathbf{A}^{\prime}$ so defined one obtains DeBYE's representation of an electromagnetic field in terms of a pair of potentials. The fields $\mathbf{A}$ and $\mathbf{A}^{\prime}$ also have the simple vector representations

$$
\mathbf{A}=\nabla \times(\nabla \times u \mathbf{r}), \quad \mathbf{A}^{\prime}=k \nabla \times v \mathbf{r},
$$

an observation which is due to Sommerfeld [22].

Equations (1.8) and (1.9) demonstrate the close relationship between the Debye potentials for an electromagnetic field (A, B) and the radial components $A_{1}, B_{1}$ of the field. In the particular case where

$$
u(r)=z_{n}(k r) S_{n}(\theta, \phi), \quad v(r)=z_{n}(k r) T_{n}(\theta, \phi),
$$

equations (1.8) and (1.9) become

$$
\begin{aligned}
& A_{1}=n(n+1) \frac{z_{n}(k r)}{r} S_{n}(\theta, \phi), \\
& B_{1}=n(n+1) \frac{z_{n}(k r)}{r} T_{n}(\theta, \phi) .
\end{aligned}
$$

This form of the relationship between the radial components and the Debye potentials has been stressed by Bouwkamp \& CAsImIR [5] in a recent paper on Debye potentials and multipole expansions.

2. Vector Wave Functions and Debye Potentials. Maxwell's equations for a 
time-harmonic electromagnetic field in a homogeneous isotropic medium may be written*

$$
\begin{aligned}
& \nabla \times \mathbf{A}=k \mathbf{B}, \\
& \nabla \times \mathbf{B}=k \mathbf{A} .
\end{aligned}
$$

The parameter $k^{2}$ may be an arbitrary non-zero complex number and $k$ is understood to be that square root of $k^{2}$ which satisfies $\operatorname{Im} k \geqq 0$. Both of the vector fields A and B satisfy the vector Helmholtz equation,

$$
\nabla \times(\nabla \times \mathbf{A})-k^{2} \mathbf{A}=0 .
$$

The author calls (2.2), rather than $\nabla^{2} \mathbf{A}+k^{2} \mathbf{A}=0$, the vector Helmholtz equation because it plays the same role in vector radiation problems that the scalar Helmholtz equation, $\nabla^{2} u+k^{2} u=0$, plays in scalar radiation problems. Notice that if A (or B) satisfies (2.2) and if B (or A) is defined by the first (or second) of equations (2.1), then the pair (A, B) satisfies (2.1). Thus the systems (2.1) and (2.2) are equivalent.

Several classes of solutions of the scalar and vector Helmholtz equations are defined next. Their introduction simplifies the statement of results in subsequent sections of the paper.

Definition 1. A complex-valued vector vector field $\mathbf{A}$ is called a vector wave function for a domain (open connected set) $D$ if it is defined and of class $C^{2}$ in $D$ and satisfies the vector Helmholtz equation (2.2) at each point of $D$.

If $(x, y, z)$ are rectangular coordinates and

$$
\mathbf{A}=A_{1} \hat{\mathbf{x}}+A_{2} \hat{\mathbf{y}}+A_{3} \hat{\mathbf{z}}
$$

is a vector wave function for $D$ then the rectangular components $A_{i}$ are of class $C^{2}$ and satisfy the scalar Helmholtz equation in $D$. It follows that the $A_{i}$ are analytic in $D$. One consequence of this is that if $\mathbf{A}$ is a vector wave function for $D$ then $\mathrm{B}=(1 / k) \nabla \times \mathrm{A}$ is also a vector wave function for $D$.

Definition 2. If $\mathbf{A}$ is a vector wave function for $D$ then the vector wave function $\mathbf{B}=(1 / k) \nabla \times \mathbf{A}$ is called the associate of $\mathbf{A}$ in $D$.

It is obvious that $\mathbf{B}$ is the associate of $\mathbf{A}$ if and only if $\mathbf{A}$ is the associate of $\mathbf{B}$. Hence it is appropriate to refer to $\mathbf{A}$ and $\mathbf{B}$ as a pair of associated vector wave functions.

Definition 3. An exterior domain $D$ is a domain that contains all points lying outside of some sphere.

Definition 4. Let $D$ be an exterior domain. A vector field $\mathbf{A}$ is a vector radiation function for $D$ if it is a vector wave function for $D$ and, in addition, satisfies

* A, B, and $k$ are related to the usual complex electric and magnetic vectors, $\mathbf{E}$ and $\mathbf{H}$, and the constitutive parameters of the medium, $\epsilon, \mu$, and $\sigma$, by the equations $\mathbf{A}=\sqrt{\sigma-i \omega \epsilon}$ $\mathbf{E}, \mathbf{B}=\sqrt{i \omega \mu} \mathrm{H}$, and $k^{2}=\omega^{2} \epsilon \mu+i \omega \mu \sigma$. The M.K.S. system of units is assumed and $\nu=\omega / 2 \pi$ is the frequency of the harmonic time dependence. 
the Silver-Müller radiation condition*

$$
\lim _{R \rightarrow \infty} \int_{S_{R}}|\hat{\mathbf{x}} \times(\nabla \times \mathbf{A})+i k \mathbf{A}|^{2} d S=0 .
$$

Here $S_{R}$ is a sphere of radius $R$ about a fixed center, $\hat{\mathbf{r}}$ is a unit normal vector to $S_{R}$, and $d S$ is the element of area on $S_{R}$.

The analogues for the scalar Helmholtz equation

$$
\nabla^{2} u+k^{2} u=0
$$

of vector wave and vector radiation functions are also needed below. Their definitions follow.

Definition 5. A complex-valued scalar function $u$ is a scalar wave function for a domain $D$ if it is defined and of class $C^{2}$ in $D$ and satisfies the scalar Helmholtz equation (2.4) at each point of $D$.

Definition 6. A function $u$ is a scalar radiation function for the exterior domain $D$ if it is a scalar wave function for $D$ and, in addition, satisfies the Sommerfeld radiation condition $\dagger$

$$
\lim _{R \rightarrow \infty} \int_{S_{R}}\left|\frac{\partial u}{\partial r}-i k u\right|^{2} d S=0
$$

Vector and scalar radiation functions are important in mathematical physics where they describe the electromagnetic and acoustic radiation (respectively) scattered by bounded scatterers. Several of the basic properties of these classes of functions have been demonstrated by the author [26, 27]. The principal result of the present paper is a representation of an arbitrary vector wave (or radiation) function in terms of a pair of scalar wave (or radiation) functions.

A complex-valued vector field $\boldsymbol{\Pi}$ that is defined in a domain $D$ and satisfies

$$
\nabla^{2} \boldsymbol{\Pi}+k^{2} \mathbf{\Pi}=\nabla \phi
$$

there, where $\phi$ is an arbitrary function, is called a Hertz vector for $D$ [22]. Equation (2.6) may be written

$$
\nabla \times(\nabla \times \mathbf{\Pi})-k^{2} \mathbf{\Pi}=\nabla(\nabla \cdot \mathbf{\Pi}-\phi),
$$

from which it is obvious that each Hertz vector (provided it is sufficiently differentiable) gives rise to a pair of associated vector wave functions, namely

* The original radiation condition of Siluver \& MÜLLER was

$$
\lim _{r \rightarrow \infty} r\{\hat{\mathbf{r}} \times(\nabla \times \mathbf{A})+i k \mathbf{A}\}=0,
$$

uniformly in $\hat{\mathbf{r}}$. The author has shown [26] that the weaker form (2.3) is sufficient for the applications and, indeed, that it implies the stronger form used by SILver \& MÜLLER.

$\uparrow$ SOMMERFELD's original radiation condition is

$$
\lim _{r \rightarrow \infty} r\left(\frac{\partial u}{\partial r}-i k u\right)=0,
$$

uniformly in $r$. The author has shown [27] that it is a consequence of the weaker condition (2.5). 


$$
\mathbf{A}=k(\nabla \times \mathbf{\Pi}) \quad \text { and } \quad \mathbf{B}=\nabla \times(\nabla \times \mathbf{\Pi}) .
$$

Now let $\psi$ be a scalar wave function for $D$, let $\mathbf{r}$ be a position vector relative to an origin lying outside $D$, and write

$$
\boldsymbol{\Pi}=\psi \mathbf{r} .
$$

Then it is easy to verify that

$$
\nabla^{2} \boldsymbol{\Pi}+k^{2} \boldsymbol{\Pi}=2 \nabla \psi
$$

Thus (2.8) defines a radial Hertz vector for $D$. The scalar wave function $\psi$ is called a Debye potential [22, p. 873] for the vector wave functions defined by (2.7). More precisely, $\psi$ is called a magnetic Debye potential for A and an electric Debye potential for B. The central theorem of this paper states that every vector wave function $\mathbf{A}$ defined in a spherical domain $a<r<b$ can be represented there as a sum,

$$
\nabla \times(\nabla \times u \mathbf{r})+k(\nabla \times v \mathbf{r}),
$$

of fields having electric and magnetic Debye potentials. A proof of this theorem that is based on a representation for fields of tangent vectors on a sphere is given below. The next section is devoted to a study of this representation.

3. A Field Representation for Spherical Domains. In the remainder of the paper the points $P$ of space are identified with their position vectors $\mathbf{r}=\overrightarrow{O P}$ relative to a fixed origin $O$. The notations $r=|\mathbf{r}|$ and and $\hat{\mathbf{r}}=\mathbf{r} / r$ are used and the unit vector $\mathbf{r}$ is often identified with the corresponding point on the unit sphere $\Omega$ about $O$. Let $R$ represent the set of positive real numbers. Then $\mathbf{r}=r \hat{\mathbf{r}}$ and the correspondence $P \leftrightarrow(r, \hat{\mathbf{r}})$ is one-to-one between the set of points of space different from $O$ and the set $R \times \Omega$. Thus it is appropriate to call $r$ and $\hat{\mathbf{r}}$ coordinates for $P$. In this paper they are called spherical coordinates for $P$ relative to $O$. The usual "geographic" coordinates $(\theta, \phi)$ for $\hat{\mathbf{r}}$ are not used because of the artificial difficulties caused by the singularities of these coordinates at the poles $\theta=0$ and $\theta=\pi$. Of course, no such difficulties arise when a local coordinate system is all that is needed and the usual spherical coordinates $(r, \theta, \phi)$ are used frequently in discussing local questions.

Every vector field $\mathbf{A}$ has an expansion

$$
\mathbf{A}=A_{1} \hat{\mathbf{r}}+\mathbf{A}_{t}
$$

where, for each point $\mathbf{r}, A_{1}(\mathbf{r})$ is a number and $\mathbf{A}_{t}(\mathbf{r})$ is a tangent vector to $\Omega$ at $\hat{\mathbf{r}}$. In this paper $A_{1}$ and $\mathbf{A}_{t}$ are called the radial and tangential components, respectively, of $\mathbf{A}$.

The existence theorem for Maxwell's equations in an exterior domain [25] implies that there is a vector wave function for $r>1$ whose tangential component on $\Omega$ equals an arbitrary continuous field of tangents to $\Omega$. Thus the theorem that every vector wave function $\mathbf{A}$ for $a<r<b$ can be written 


$$
\mathbf{A}=\nabla \times(\nabla \times u \mathbf{r})+k \nabla \times v \mathbf{r}
$$

suggests that the tangential component of the right side of (3.2) is sufficiently general to represent an arbitrary field of tangents to $\Omega$. This result is, in essence, correct and a theorem to this effect is stated and proved below. It is then shown to lead, in a natural way, to a proof of the existence of the Debye potentials $u$ and $v$.

Several differential invariants connected with the sphere $\Omega$ are important in the study of the representation (3.2) in spherical coordinates. The fundamental ones are the gradient $\nabla_{t} f$ of a scalar function $f$ and the divergence $\nabla_{t} \cdot \boldsymbol{\alpha}$ of a field $\boldsymbol{\alpha}$ of tangent vectors on $\Omega$. In geographic coordinates $(\theta, \phi)$ these operators take the forms

$$
\nabla_{t} f=\frac{\partial f}{\partial \theta} \hat{\boldsymbol{\theta}}+\frac{1}{\sin \theta} \frac{\partial f}{\partial \phi} \hat{\boldsymbol{\phi}}
$$

and

$$
\nabla_{t} \cdot \boldsymbol{\alpha}=\frac{1}{\sin \theta} \frac{\partial}{\partial \theta}\left(\sin \theta \alpha_{1}\right)+\frac{1}{\sin \theta} \frac{\partial \alpha_{2}}{\partial \phi}
$$

where

$$
\boldsymbol{\alpha}=\alpha_{1} \hat{\boldsymbol{\theta}}+\alpha_{2} \hat{\boldsymbol{\phi}} .
$$

These operators can be defined by (3.3) and (3.4) and then can be shown to be invariants by the usual methods of tensor calculus*. Several other invariants that are derived from (3.3) and (3.4) occur frequently in our analysis. They are $\nabla_{t}^{2} f=\nabla_{t} \cdot \nabla_{t} f$, which is Beltrami's operator (the second differential parameter) for $\Omega, \hat{\mathrm{r}} \times \nabla_{t} f$, and $\nabla_{t} \cdot(\hat{\mathbf{r}} \times \boldsymbol{\alpha})$. The differential identity

$$
\nabla_{t} \cdot\left(\mathbf{r} \times \nabla_{t} f\right)=0
$$

and the algebraic identity

$$
\hat{\mathbf{r}} \times(\hat{\mathbf{r}} \times \boldsymbol{\alpha})=-\boldsymbol{\alpha}
$$

are also used in the calculations.

Now let us consider the representation (3.2). A short vector calculation shows that

$$
\nabla \times v \mathbf{r}=\nabla_{t} v \times \hat{\mathbf{r}}
$$

A similar calculation gives

$$
\nabla \times(\nabla \times u \mathbf{r})=\left(-\frac{1}{r} \nabla_{t}^{2} u\right) \hat{\mathbf{r}}+\nabla_{t} \frac{1}{r} \frac{\partial(r u)}{\partial r}
$$

for all functions $u$ of class $C^{2}$. On combining these results it is seen that if A

* Note, however, that the components of $\alpha$ and $\nabla_{t} f$ given above are the physical ones, not the covariant or contravariant ones usually used in tensor analysis. 
is defined by (3.2) then

$$
\mathbf{A}=\left(-\frac{1}{r} \nabla_{t}^{2} u\right) \hat{\mathbf{r}}+\nabla_{t} \frac{1}{r} \frac{\partial(r u)}{\partial r}-k \hat{\mathbf{r}} \times \nabla_{t} v .
$$

Hence $A_{1}=-(1 / r) \nabla_{t}^{2} u$ and

$$
\mathbf{A}_{t}=\nabla_{t} S+\hat{\mathbf{r}} \times \nabla_{t} T
$$

where $S=r^{-1} \partial(r u) / \partial r$ and $T=-k v$. The differential operations in the expression for $A_{t}$ refer to $\Omega$ only and, if our conjecture is correct, "every" field $\alpha$ of tangent vectors on $\Omega$ should be representable in this form:

$$
\boldsymbol{\alpha}=\nabla_{t} S+\hat{\mathbf{r}} \times \nabla_{t} T .
$$

Let us investigate the extent to which this last statement is true. If $\boldsymbol{\alpha}$ is given by (3.9) then the identities (3.6) and (3.7) imply

$$
\nabla_{t}^{2} S=\nabla_{t} \cdot \boldsymbol{\alpha} \quad \text { and } \quad \nabla_{t}^{2} T=-\nabla_{t} \cdot(\hat{\mathbf{r}} \times \boldsymbol{\alpha})
$$

and these may be regarded as differential equations for $S$ and $T$. This observation provides a key to a proof of the existence of the representation (3.9). To justify this assertion it is shown below that equations (3.10) have integrals $S$ and $T$ and that the functions $\nabla_{t} \cdot \boldsymbol{\alpha}$ and $\nabla_{t} \cdot(\hat{\mathbf{r}} \times \boldsymbol{\alpha})$ determine $\boldsymbol{\alpha}$ uniquely. These results are then combined to yield the existence proof.

Equations (3.10) have the form

$$
\nabla_{t}^{2} u=f
$$

where $f$ is a given function on $\Omega$. The integration of this equation has been studied by Zermelo [29], Hadamard [11], Hilbert [12], and, more recently, by BIDAL \& DE RHAM [2]*. Their results are summarized in the following theorem.

Theorem 3.1. Let $f$ be of class $C^{2}$ on $\Omega$. Then there exists a solution $u$ of

$$
\nabla_{t}^{2} u=f
$$

if and only if

$$
\int_{\Omega} f d \Omega=0
$$

The solution $u$, when it exists, is of class $C^{2}$.

Corollary 3.1. If $f$ satisfies (3.12) then (3.11) has a unique solution $u$ for which

$$
\int_{\Omega} u d \Omega=0
$$

* Bidal \& DE RHAM studied the integration of $\Delta \mu=\beta$ where $\mu$ and $\beta$ are fields of $p$-vectors on an $n$-dimensional Riemannian manifold and $\Delta$ is a generalization of the Laplacian. The present theorem is the special case of theirs that results when $n=2, p=0$, and the manifold is the unit sphere $\Omega$. 
This is a consequence of the well known result that the only solution of $\nabla_{t}^{2} u=0$ is a constant [8, p. 512].

Corollary 3.2. If $f$ satisfies (3.12) then the unique solution of (3.11) satisfying (3.13) is given by the formula

$$
u(\hat{\mathbf{x}})=\frac{1}{2 \pi} \int_{\Omega}(\log \sin \gamma / 2) f\left(\hat{\mathbf{r}}^{\prime}\right) d \Omega^{\prime}
$$

where $\gamma=\gamma\left(\hat{\mathbf{r}}, \hat{\mathbf{r}}^{\prime}\right)$ is the geodesic distance from $\hat{\mathbf{r}}$ to $\hat{\mathbf{r}}^{\prime}$ on $\Omega$.

This result was discovered by ZermeLo [28]. It is not difficult to verify directly that the function $u$ defined by (3.14) satisfies (3.11) and (3.13) when $f$ satisfies (3.12).

The following theorem is employed to show that $\nabla_{t} \cdot \boldsymbol{\alpha}$ and $\nabla_{t} \cdot(\hat{\mathbf{r}} \times \boldsymbol{\alpha})$ determine $\alpha$ uniquely. It was discovered by Sir Horace LAmb and was published in 1884 in a paper on the motion of a viscous fluid in a spherical domain [17].

Theorem 3.2. Let $\mathbf{A}$ be a vector field that is defined and of class $C^{1}$ in a domain $a<r<b$, and suppose that

$$
\begin{aligned}
\nabla \cdot \mathbf{A} & =0, \\
\mathbf{r} \cdot \mathbf{A} & =\mathbf{0},
\end{aligned}
$$

and

$$
\mathbf{r} \cdot(\nabla \times \mathbf{A})=0 \text { in } a<r<b .
$$

Then A must vanish identically in $a<r<b$.

Condition (b) implies that $\mathbf{A}=\mathbf{A}_{t}=\boldsymbol{\alpha}$ is a field of tangent vectors on each sphere $r=$ constant $a<r<b$. Condition (a) then reduces to $\nabla_{\mathfrak{t}} \cdot \boldsymbol{\alpha}=0$. Moreover, an examination of the expression for $\nabla \times \mathbf{A}$ in spherical coordinates shows that (c) is equivalent to the condition $\nabla_{t} \cdot(\hat{\mathbf{r}} \times \boldsymbol{\alpha})=0$. Thus Lamb's theorem may be reduced to the following one.

Theorem 3.3. Let $\alpha$ be a field of tangent vectors on $\Omega$ that is of class $C^{1}$ and satisfies

$$
\nabla_{t} \cdot \boldsymbol{\alpha}=0
$$

and

$$
\nabla_{\imath} \cdot(\hat{\mathbf{r}} \times \boldsymbol{\alpha})=0
$$

there. Then $\boldsymbol{\alpha}=0$ on $\Omega$.

Proof. We notice that (b) implies the existence of a function $F=F(\hat{\mathbf{r}})$, defined, single-valued, and of class $C^{2}$ on $\Omega$, for which

$$
\boldsymbol{\alpha}=\nabla_{t} F .
$$


To prove this it is sufficient to show that if $\Gamma$ is any closed oriented piecewise smooth simple curve on $\Omega$ and $\hat{\mathrm{t}}$ is a unit tangent vector to it then

$$
\int_{\Gamma} \boldsymbol{\alpha} \cdot \hat{\mathbf{t}} d s=0
$$

But, because $\Omega$ is simply connected, $\Gamma$ is homologous to a sum of closed curves $\Gamma_{i}$ having the same properties as $\Gamma$ and enclosing regions $R_{i}$ whose (spherical) diameter is less than $\pi . R_{i}$ can be covered by a geographic coordinate system $(\theta, \phi)$ whose poles are outside the region. Then (b) implies that if $\alpha=\alpha_{1} \hat{\boldsymbol{\theta}}+\alpha_{2} \hat{\boldsymbol{\phi}}$ in $R_{i}$,

$$
\frac{\partial}{\partial \theta}\left(\sin \theta \alpha_{2}\right)-\frac{\partial \alpha_{1}}{\partial \phi}=0
$$

throughout $R_{i}$. Hence

$$
d F=\alpha_{1} d \theta+\sin \theta \alpha_{2} d \phi
$$

is exact in $R_{\boldsymbol{i}}$ and so $\int_{\Gamma_{\boldsymbol{i}}} \boldsymbol{\alpha} \cdot \hat{\mathbf{t}} d s=0$, which implies (3.15). Finally, condition (a) implies that $F$ satisfies

$$
\nabla_{t}^{2} F=0
$$

on $\Omega$, and this equation is known to be satisfied only by a constant function. Thus $\boldsymbol{\alpha}=0$, as was to be shown.

The above proof is, in principle, very similar to LAmB's original proof. However, he used a single system of geographic coordinates $(\theta, \phi)$, which produces difficulties due to the presence of the singular points $\theta=0$ and $\theta=\pi$. Theorems 3.1 and 3.3 enable us to prove the following theorem concerning the representation (3.9).

Theorem 3.4. Let $\boldsymbol{\alpha}$ be a field of tangent vectors on $\Omega$ that is of class $C^{3}$ there. Then there exist unique functions $S$ and $T$ that are defined and of class $C^{2}$ on $\Omega$ and satisfy

$$
\int_{\Omega} S d \Omega=\int_{\Omega} T d \Omega=0
$$

and

$$
\boldsymbol{\alpha}=\nabla_{t} S+\hat{\mathbf{r}} \times \nabla_{t} T
$$

on $\Omega$.

Proof. To begin, the functions $f=\nabla_{t} \cdot \boldsymbol{\alpha}$ and $g=1-\nabla_{t} \cdot(\hat{\mathbf{r}} \times \boldsymbol{\alpha})$ are of class $C^{2}$ on $\Omega$ and satisfy (3.12), the latter being a consequence of the divergence theorem for $\Omega$. Hence there exist unique functions $S$ and $T$ that satisfy (a) and equations (3.10), by Theorem 3.1 and Corollary 3.1. If

$$
\boldsymbol{\alpha}=\nabla_{t} S+\hat{\mathbf{r}} \times \nabla_{t} T+\hat{\beta},
$$


where $S$ and $T$ are these integrals, then

$$
\nabla_{t} \cdot \hat{\beta}=0 \text { and } \nabla_{t} \cdot(\hat{\mathbf{r}} \times \hat{\boldsymbol{\theta}})=0
$$

on $\Omega$, by the identities (3.6) and (3.7) and equations (3.10). But then, since $\beta$ is clearly of class $C^{1}$ on $\Omega, \beta=0$ by Theorem 3.3, which gives (b).

It is of interest to note that Theorems 3.3 and 3.4 are special cases of theorems of Hodge concerning fields of $p$-vectors on a Riemannian manifold. HodGE's decomposition theorem [2] states that every field $\boldsymbol{\alpha}$ of $p$-vectors on a closed orientable $n$-dimensional Riemannian manifold $M$ has a unique decomposition into three parts,

$$
\boldsymbol{\alpha}=\boldsymbol{\alpha}_{1}+\boldsymbol{\alpha}_{2}+\alpha_{3},
$$

where $\boldsymbol{\alpha}_{1}$ is exact $\left(\boldsymbol{\alpha}_{1}=d \boldsymbol{\beta}\right), \boldsymbol{\alpha}_{2}$ is coexact $\left(\boldsymbol{\alpha}_{2}=\delta \gamma\right)$, and $\boldsymbol{\alpha}_{3}$ is harmonic $\left(d \boldsymbol{\alpha}_{3}=0\right.$, $\left.\delta \boldsymbol{\alpha}_{3}=0\right)$. HoDGE's existence theorem for harmonic $p$-vector fields [2] states that the number of linearly independent harmonic $p$-vector fields on $M$ equals the $p$-th Betti number of $M$. The decomposition (b) of Theorem 3.4 is Hodge's decomposition for a field $\alpha$ of 1 -vectors on the 2 -dimensional manifold $\Omega$. This is easily verified by noting that $\nabla_{t} S$ corresponds to the 1 -form $d S$ and $\hat{\mathbf{r}} \times \nabla_{t} T$ corresponds to the 1-form $\delta(T d \Omega)$, in the notation of BIDAL \& DE RHAM [2]. Thus $\nabla_{t} S$ and $\hat{\mathbf{r}} \times \nabla_{t} T$ correspond to the parts $\boldsymbol{\alpha}_{1}$ and $\boldsymbol{\alpha}_{2}$, respectively, of HoDGE's decomposition. The term $\boldsymbol{\alpha}_{3}$ is absent because the first Betti number of $\Omega$ is zero. Thus Theorem 3.3 exemplifies HodGE's existence theorem.

4. The Existence Theorem for Debye Potentials. In this section it is shown that the results of $\S 3$ lead to a simple and direct proof of the existence of Debye potentials for vector wave functions defined in spherical domains, and several corollaries of the existence theorem are derived. First, let us consider a vector field A that is defined and of class $C^{3}$ in a domain $a<r<b$. Theorem 3.4 implies that

$$
\mathbf{A}=R \hat{\mathbf{r}}+\nabla_{t} S+\hat{\mathbf{r}} \times \nabla_{t} T
$$

on each sphere $r=r_{0}$ with $a<r_{0}<b$. Thus (4.1) holds throughout $a<r<b$ and $R, S$, and $T$ are functions of $r$ and $\hat{\mathrm{r}}$. $R, S$, and $T$ have many of the properties associated with a set of components for the vector $\mathbf{A}$. Thus they are linear functions of $\mathbf{A}$, and $\mathbf{A}=0$ if and only if $R=S=T=0$, by Theorem 3.4. In this paper $R, S$, and $T$ are called the global spherical components (or, more briefly, global components) to distinguish them from the components $A_{1}, A_{2}$, and $A_{3}$ defined by

$$
\mathbf{A}=A_{1} \hat{\mathbf{r}}+A_{2} \hat{\boldsymbol{\theta}}+A_{3} \hat{\boldsymbol{\phi}} .
$$

$R$ and $A_{1}$ are, of course, the same. An important difference between the global components and the usual spherical components for a field vector $\mathbf{A}$ at a point $P_{0}$ is that the former are not defined unless $\mathbf{A}$ is defined on the whole sphere $r=r_{0}$ through $P_{0}$, while A needs to be defined only at $P_{0}$ to have a representation (4.2). 
Now suppose that $\mathbf{A}$ is analytic in $a<r<b$. It follows that the global components of $\mathbf{A}$ are also analytic there. This is obviously true of $R=\hat{\mathbf{r}} \cdot \mathbf{A}$. To prove it for $S$ let $P_{0}$ be any point in $a<r<b$ and introduce spherical coordinates $(r, \theta, \phi)$ whose axis does not pass through $P_{0}$. Then $S$ satisfies

$$
\frac{1}{\sin \theta} \frac{\partial}{\partial \theta}\left(\sin \theta \frac{\partial S}{\partial \theta}\right)+\frac{1}{\sin ^{2} \theta} \frac{\partial^{2} S}{\partial \phi^{2}}=f(r, \theta, \phi)
$$

near $P_{0}$, where $f=\nabla_{t} \cdot \mathbf{A}$ is analytic. Under the analytic transformation

$$
\psi=\log \tan \frac{\theta}{2}, \quad \phi=\phi
$$

equation (4.3) becomes

$$
\frac{\partial^{2} S}{\partial \psi^{2}}+\frac{\partial^{2} S}{\partial \phi^{2}}=F(r, \psi, \phi)
$$

where $F(r, \psi, \phi)=\operatorname{sech}^{2} \psi f(r, \theta, \phi)$ is analytic. $S$ is also known to be of class $C^{2}$, by Theorem 3.4, and it follows from a well known result for elliptic partial differential equations that $S$ is analytic near $P_{0}$ (see PrCARD [21] or Courant-Hilbert [9], II, pp. 339-342). The same argument shows that $T$ is analytic. In particular, if $\mathbf{A}$ is analytic then the components $R$ and $A_{t}=$ $\nabla_{t} S+\hat{\mathbf{r}} \times \nabla_{t} T$ in (4.1) may be differentiated any number of times.

The usual expression for $\nabla \times \mathbf{A}$ in spherical coordinates may be written (see e.g. [18, p. 146])

$$
\nabla \times \mathbf{A}=\left(-\frac{1}{r} \nabla_{t} \cdot \hat{\mathbf{r}} \times \mathbf{A}_{t}\right) \hat{\mathbf{r}}+\frac{1}{r} \frac{\partial}{\partial r}\left(r \hat{\mathbf{r}} \times \mathbf{A}_{t}\right)-\hat{\mathbf{r}} \times \nabla_{t} \frac{R}{r} .
$$

When the global components $S$ and $T$ are introduced (4.5) becomes

$$
\nabla \times \mathbf{A}=\left(\frac{1}{r} \nabla_{t}^{2} T\right) \hat{\mathbf{r}}-\nabla_{t}\left(\frac{1}{r} \frac{\partial(r T)}{\partial r}\right)+\hat{\mathbf{r}} \times \nabla_{t} \frac{1}{r}\left(\frac{\partial(r S)}{\partial r}-R\right),
$$

and this has the form

$$
\nabla \times \mathbf{A}=R^{\prime} \hat{\mathbf{r}}+\nabla_{t} S^{\prime}+\hat{\mathbf{r}} \times \nabla_{t} T^{\prime}
$$

Thus

$$
\begin{aligned}
& R^{\prime}=\frac{1}{r} \nabla_{t}^{2} T \\
& S^{\prime}=-\frac{1}{r} \frac{\partial(r T)}{\partial r}, \quad \text { and } \\
& T^{\prime}=\frac{1}{r}\left(\frac{\partial(r S)}{\partial r}-R+\frac{1}{4 \pi} \int_{\Omega} R d \Omega\right),
\end{aligned}
$$

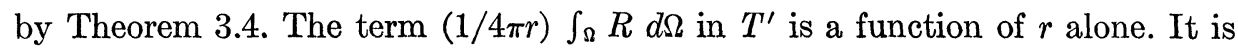
included to make $\int_{\Omega} T^{\prime} d \Omega=0$ and contributed nothing to $\nabla \times \mathbf{A}$. 
Now suppose that $\mathbf{A}$ is a vector wave function for a domain $a<r<b$. Then $R, S$, and $T$ are analytic in $a<r<b$ and the expressions (4.6) for the global components of $k \mathbf{B}=\nabla \times \mathbf{A}$ are valid. Let us see what the vector Helmholtz equation, which is satisfied by $\mathbf{A}$, implies for the global components $R, S$, and $T$. First,

$$
\int_{\Omega} R d \Omega=\int_{\Omega} \hat{\mathbf{r}} \cdot \mathbf{A} d \Omega=\frac{1}{k} \int_{\Omega} \hat{\mathbf{r}} \cdot(\nabla \times \mathbf{B}) d \Omega
$$

where B is the associate of A. Thus $\int_{\Omega} R d \Omega=0$ by Stokes' theorem, because $\hat{\mathrm{r}}$ is the unit normal to $\Omega$ and $\Omega$ is closed. Second, two applications of the formulas (4.6) show that the vector Helmholtz equation for $\mathbf{A}$ is equivalent to the three scalar equations

$$
\begin{aligned}
& k^{2} R=\frac{1}{r^{2}} \nabla_{t}^{2}\left\{\frac{\partial(r S)}{\partial r}-R\right\}, \\
& k^{2} S=-\frac{1}{r} \frac{\partial}{\partial r}\left\{\frac{\partial(r S)}{\partial r}-R\right\}, \quad \text { and } \\
& k^{2} T=-\frac{1}{r} \frac{\partial^{2}(r T)}{\partial r^{2}}-\frac{1}{r^{2}} \nabla_{t}^{2} T .
\end{aligned}
$$

The last equation is the scalar Helmholtz equation $\nabla^{2} T+k^{2} T=0$ in spherical coordinates. To see the meaning of the first two equations of (4.7) write

$$
u=\frac{1}{k^{2} r}\left\{R-\frac{\partial(r S)}{\partial r}\right\} \text {. }
$$

Then

$$
R=-\frac{1}{r} \nabla_{t}^{2} u \quad \text { and } \quad S=\frac{1}{r} \frac{\partial(r u)}{\partial r} .
$$

On multiplying the last equation by $r$, differentiating it with respect to $r$, and subtracting it from the first equation we find

$$
k^{2} u=-\frac{1}{r^{2}} \nabla_{t}^{2} u-\frac{1}{r} \frac{\partial^{2}(r u)}{\partial r^{2}}
$$

that is, $u$ also satisfies the scalar Helmholtz equation. Thus both $u$ and $v=-k^{-1} T$ are scalar wave functions for $a<r<b$ and, by (4.8),

$$
\mathbf{A}=\left(-\frac{1}{r} \nabla_{t}^{2} u\right) \hat{\mathbf{r}}+\nabla_{t} \frac{1}{r} \frac{\partial(r u)}{\partial r}-k \hat{\mathbf{r}} \times \nabla_{t} v .
$$

It was shown in $\S 3$ that this equation is equivalent to

$$
\mathbf{A}=\nabla \times(\nabla \times u \mathbf{r})+k \nabla \times v \mathbf{r} .
$$

Thus Theorem 3.4 is seen to lead directly to a representation of $\mathbf{A}$ by Debye potentials. Our result may be stated as follows: 
Theorem 4.1. Let A and B be associated vector wave functions for a domain $a<r<b$. Then there exist unique functions $u$ and $v$ such that

(a) $u$ and $v$ are scalar wave functions for $a<r<b$,

(b) $\int_{\Omega} u(r \hat{\mathbf{r}}) d \Omega=\int_{\Omega} v(r \hat{\mathbf{r}}) d \Omega=0$ on $a<r<b$, and

(c) $\mathbf{A}=\nabla \times(\nabla \times u \mathbf{r})+k \nabla \times v \mathbf{r}$ and

$\mathbf{B}=\nabla \times(\nabla \times v \mathbf{r})+k \nabla \times u \mathbf{r}$, on $a<r<b$.

Proof. The existence of functions $u$ and $v$ satisfying (a), (b), and (c) has been shown to follow from Theorem 3.4. The representation for B may be obtained by taking the curl of the representation for $\mathbf{A}$. To prove uniqueness let $\left(u^{\prime}, v^{\prime}\right)$ and $\left(u^{\prime \prime}, v^{\prime \prime}\right)$ be two pairs satisfying (a), (b), and (c). Then $u=u^{\prime}-u^{\prime \prime}$ and $v=v^{\prime}-v^{\prime \prime}$ are Debye potentials for the zero fields $\mathbf{A}=\mathbf{B}=0$. It follows that

$$
\nabla_{t} \frac{1}{r} \frac{\partial(r u)}{\partial r}-k \hat{\mathrm{r}} \times \nabla_{t} v=0 \quad \text { and } \quad \nabla_{t} \frac{1}{r} \frac{\partial(r v)}{\partial r}-k \hat{\mathrm{r}} \times \nabla_{t} u=0 .
$$

This implies that $u=v=0$ by Theorem 3.4, since $u$ and $v$ satisfy (b).

The scalar wave functions $u$ and $v$ are called, respectively, the electric and magnetic Debye potentials for A. One consequence of Theorem 4.1 is that the electric (magnetic) Debye potential for $\mathbf{A}$ is the magnetic (electric) Debye potential for the associated vector wave function $\mathbf{B}$, and conversely. The proofs of Theorems 3.4 and 4.1 also provide explicit relationships among $\mathbf{A}, \mathbf{B}, u$, and $v$. These may be stated as follows:

Corollary 4.1. The Debye potentials $u$ and $v$ for $\mathbf{A}$ are related to the radial components $A_{1}=\hat{\mathbf{r}} \cdot \mathbf{A}$ and $B_{1}=\hat{\mathbf{r}} \cdot \mathbf{B}$ by the relations

$$
\nabla_{t}^{2} u=-r A_{1} \quad \text { and } \quad \nabla_{t}^{2} v=-r B_{1} \quad \text { on } \quad a<r<b
$$

and the reciprocal relations (Theorem 3.1, Corollary 3.2)

$$
\begin{aligned}
& u(r \hat{\mathbf{r}})=-\frac{r}{2 \pi} \int_{\Omega}\left(\log \sin \frac{\gamma}{2}\right) A_{1}\left(r \hat{\mathbf{r}}^{\prime}\right) d \Omega^{\prime} \quad \text { and } \\
& v(r \hat{\mathbf{r}})=-\frac{r}{2 \pi} \int_{\Omega}\left(\log \sin \frac{\gamma}{2}\right) B_{1}\left(r \hat{\mathbf{r}}^{\prime}\right) d \Omega^{\prime} \quad \text { on } \quad a<r<b .
\end{aligned}
$$

The differential equations (4.9) for $u$ and $v$ were demonstrated in $\S 3$, equation (3.8). The formulas (4.10) suggest an alternative proof of the existence of Debye potentials. One could define $u$ and $v$ by (4.10) and then verify that they are scalar wave functions and that the field $\nabla \times(\nabla \times u \mathbf{r})+k \nabla \times v \mathbf{r}$ coincides with the given vector wave function $\mathbf{A}$. However, the verification requires considerable analysis and is, therefore, less satisfactory than the direct approach developed above.

The vector wave functions $\mathbf{A}$ occurring in the usual boundary value problems for exterior domains satisfy the vector radiation condition (2.3), and it is natural to ask for equivalent conditions at infinity on the Debye potentials $u$ and $v$ for $\mathbf{A}$. The known existence and uniqueness theorem for scalar wave functions in ex- 
terior domains utilize SommeRFeLd's radiation condition (2.5), and we are led to hope that it may be an appropriate equivalent. Corollary 4.2 is a statement of this equivalence.

Corollary 4.2. Let $\mathbf{A}$ be a vector wave function for a domain $r>a$. Then $\mathbf{A}$ is a vector radiation function for $r>a$ if and only if the corresponding Debye potentials $u$ and $v$ are scalar radiation functions for $r>a$.

Proof. We must show that $\mathbf{A}$ satisfies the vector radiation condition if and only if $u$ and $v$ satisfy the scalar radiation condition. Now it is known [26] that if $\mathbf{A}$ satisfies the vector radiation condition then $r A_{1}$ and $r B_{1}$ satisfy the scalar radiation condition. This fact together with the formulas (4.10) for $u$ and $v$ imply that the latter also satisfy the scalar radiation condition. The necessary interchange of differentiation and integration is easy to justify (see e.g. [13, p. 356]).

To prove the converse write A and B in terms of the Debye potentials in the form (see (3.8))

$$
\begin{aligned}
& \mathbf{A}=\left(-\frac{1}{r} \nabla_{t}^{2} u\right) \hat{\mathbf{r}}+\nabla_{t}\left(\frac{1}{r} \frac{\partial(r u)}{\partial r}\right)-k \hat{\mathbf{r}} \times \nabla_{t} v, \\
& \mathbf{B}=\left(-\frac{1}{r} \nabla_{t}^{2} v\right) \hat{\mathbf{r}}+\nabla_{t}\left(\frac{1}{r} \frac{\partial(r v)}{\partial r}\right)-k \hat{\mathbf{r}} \times \nabla_{t} u
\end{aligned}
$$

Then

$$
\begin{aligned}
r\{\hat{\mathbf{r}} \times(\nabla \times \mathbf{A}) & +i k \mathbf{A}\}=k r\{\hat{\mathbf{r}} \times \mathbf{B}+i \mathbf{A}\} \\
=k & {\left[\left(-i \nabla_{t}^{2} u\right) \hat{\mathbf{r}}+i \nabla_{t}\left\{\frac{\partial(r u)}{\partial r}-i k r u\right\}+r \times \nabla_{t}\left\{\frac{\partial(r v)}{\partial r}-i k r v\right\}\right] . }
\end{aligned}
$$

Now the expansion theorem for scalar radiation functions [27] yields the expansions

$$
u=\frac{e^{i k r}}{r} \sum_{n=0}^{\infty} \frac{u_{n}(\hat{\mathbf{r}})}{r^{n}}, \quad \frac{\partial(r u)}{\partial r}-i k r u=e^{i k r} \sum_{n=1}^{\infty} \frac{n u_{n}(\hat{\mathbf{r}})}{r^{n+1}},
$$

with analogous expansions for $v$, and these series may be differentiated termwise with respect to the angular variables [27]. Moreover, the resulting series are all uniformly convergent in any region $r \geqq a+\epsilon>a$ [27]. Thus

$$
r\{\hat{\mathbf{r}} \times(\nabla \times \mathbf{A})+i k \mathbf{A}\}=0\left(\frac{1}{r}\right), \quad r \rightarrow \infty,
$$

uniformly in $\hat{\mathbf{r}}$, which implies the vector radiation condition for $\mathbf{A}$.

Theorem 3.4 can provide a representation by potentials for fields that satisfy other vector differential equations than (2.2). An example of this is furnished by time-harmonic electromagnetic fields in a spherically stratified medium. These satisfy

$$
\nabla \times \mathbf{A}=m \mathbf{B}, \quad \nabla \times \mathbf{B}=n \mathbf{A},
$$


where $m$ and $n$ are functions of $r$, the distance from a fixed point. The reasoning used to derive Theorem 4.1 may be used to prove the following theorem for solutions of (4.11).

Theorem 4.2. Let A and B be of class $C^{2}$ and satisfy (4.11) in $a<r<b$, where $m$ and $n$ are analytic and different from zero in $a<r<b$. Then there exist unique functions $u$ and $v$ such that

$$
\nabla^{2} u+(m n) u-\left(\frac{1}{n r} \frac{d n}{d r}\right) \frac{\partial(r u)}{\partial r}=0
$$

$$
\nabla^{2} v+(m n) v-\left(\frac{1}{m r} \frac{d m}{d r}\right) \frac{\partial(r v)}{\partial r}=0
$$

$$
\int_{\Omega} u(r \hat{\mathbf{r}}) d \Omega=\int_{\Omega} v(r \hat{\mathbf{r}}) d \Omega=0
$$

and

$$
\begin{aligned}
& \mathbf{A}=\frac{1}{n} \nabla \times(\nabla \times u \mathbf{r})+\nabla \times v \mathbf{r}, \\
& \mathbf{B}=\frac{1}{m} \nabla \times(\nabla \times v \mathbf{r})+\nabla \times u \mathbf{r}
\end{aligned}
$$

in $a<r<b$.

Notice that equations (a) for $u$ and $v$ are separable in spherical coordinates. Theorem 3.4 will provide a representation by potentials even if $m$ and $n$ are functions of the other coordinates, but the analogues of equations (a) will not be separable.

5. Multiple Expansions for Vector Radiation Functions. The vector fields

$$
\begin{aligned}
& \mathbf{E}_{n}(\mathbf{r})=\nabla \times\left(\nabla \times h_{n}(k r) S_{n}(\hat{\mathbf{r}}) \mathbf{r}\right), \\
& \mathbf{H}_{n}(\mathbf{r})=k \nabla \times h_{n}(k r) T_{n}(\hat{\mathbf{r}}) \mathbf{r},
\end{aligned}
$$

where

$$
h_{n}(x)=\sqrt{\frac{\pi}{2 x}} H_{n+\frac{1}{2}}^{(1)}(x)
$$

and $S_{n}$ and $T_{n}$ are spherical harmonics of order $n$, are clearly vector radiation functions for the domain $r>0 . \mathrm{E}_{n}$ and $\mathrm{H}_{n}$ are called electromagnetic multipoles of order $n$ and of electric and magnetic type, respectively. The purpose of this section is to show that every vector radiation function $\mathbf{A}$ defined in a domain $r>c$ can be expanded there in a series

$$
\mathrm{A}=\mathrm{E}_{1}+\mathrm{E}_{2}+\cdots+\mathrm{H}_{1}+\mathrm{H}_{2}+\cdots
$$

of electromagnetic multipoles. Before turning to the proof of this theorem we remark that the expansion (5.2), provided it converges uniformly, is unique. This follows from the fact that the multipoles are orthogonal on any sphere 
concentric with the origin. To see this notice that the multipoles (5.1) may be written

$$
\begin{aligned}
& \mathbf{E}_{n}=\frac{n(n+1) h_{n}(k r)}{r} S_{n}(\hat{\mathbf{r}}) \hat{\mathbf{r}}+\frac{1}{r} \frac{d\left(r h_{n}(k r)\right)}{d r} \nabla_{t} S_{n}(\hat{\mathbf{r}}), \\
& \mathbf{H}_{n}=-k h_{n}(k r) \hat{\mathbf{r}} \times \nabla_{t} T_{n}(\hat{\mathbf{r}}),
\end{aligned}
$$

or

$$
\begin{aligned}
\mathbf{E}_{n} & =A_{n} S_{n} \hat{\mathbf{1}}+B_{n} \nabla_{t} S_{n}, \\
\mathbf{H}_{n} & =C_{n}\left(\hat{\mathbf{r}} \times \nabla_{t} T_{n}\right),
\end{aligned}
$$

where $A_{n}, B_{n}$, and $C_{n}$ are functions of $r$ only. Thus

$$
\begin{aligned}
\mathbf{E}_{n} \cdot \overline{\mathrm{E}}_{m} & =\left(A_{n} \bar{A}_{m}\right) S_{n} \bar{S}_{m}+\left(B_{n} \bar{B}_{m}\right) \nabla_{t} S_{n} \cdot \nabla_{t} \bar{S}_{m}, \\
\mathbf{H}_{n} \cdot \overline{\mathrm{H}}_{m} & =\left(C_{n} \bar{C}_{m}\right)\left(\hat{\mathbf{r}} \times \nabla_{t} T_{n}\right) \cdot\left(\hat{\mathbf{r}} \times \nabla_{t} \bar{T}_{m}\right)=\left(C_{n} \bar{C}_{m}\right) \nabla_{t} T_{n} \cdot \nabla_{t} \bar{T}_{m}, \\
\mathbf{E}_{n} \cdot \overline{\mathrm{H}}_{m} & =\left(B_{n} \bar{C}_{m}\right) \hat{\mathbf{r}} \cdot \nabla_{t} \bar{T}_{m} \times \nabla_{t} S_{n} .
\end{aligned}
$$

The spherical harmonics $S_{n}$ are known to be orthogonal on $\Omega$. Green's theorem for a region $\Sigma$ on $\Omega$ bounded by a curve $\Gamma$ may be written (see [3, p. 174])

$$
\int_{\Sigma} u \nabla_{t}^{2} v d \Omega+\int_{\Sigma} \nabla_{t} u \cdot \nabla_{t} v d \Omega=\int_{\Gamma} u \frac{\partial v}{\partial n} d s .
$$

If $\Sigma=\Omega$ then $\Gamma$ vanishes. Hence, taking $u=S_{n}, v=\bar{S}_{m}$,

$$
\int_{\Omega} \nabla_{t} S_{n} \cdot \nabla_{t} \bar{S}_{m} d \Omega=m(m+1) \int_{\Omega} S_{n} \bar{S}_{m} d \Omega,
$$

since $\nabla_{t}^{2} S_{m}=-m(m+1) S_{m}$. These equations prove that

$$
\int_{\Omega} \mathbf{E}_{n} \cdot \overline{\mathrm{E}}_{m} d \Omega=0, \quad \int_{\Omega} \mathbf{H}_{n} \cdot \overline{\mathbf{H}}_{m} d \Omega=0, \quad n \neq m,
$$

for any value of $r$. The integral $\int_{\Omega} \mathbf{E}_{n} \cdot \overline{\mathbf{H}}_{m} d \Omega$ can be shown to vanish by means of the following lemma.

Lemma 5.1. Let $f(\hat{\mathbf{r}})$ and $g(\hat{\mathbf{r}})$ be single-valued and of class $C^{2}$ on the unit sphere $\Omega$. Then

$$
\int_{\Omega} \hat{\mathbf{r}} \cdot \nabla_{t} f \times \nabla_{t} g d \Omega=0 .
$$

Proof. Introduce geographic coordinates $(\theta, \phi)$ on $\Omega$. Then

Moreover,

$$
\hat{\mathbf{r}} \times \nabla_{t} f \times \nabla_{t} g=\frac{1}{\sin \theta}\left(\frac{\partial f}{\partial \theta} \frac{\partial g}{\partial \phi}-\frac{\partial f}{\partial \phi} \frac{\partial g}{\partial \theta}\right) .
$$

$$
\int_{0}^{2 \pi} \frac{\partial f}{\partial \theta} \frac{\partial g}{\partial \phi} d \phi=-\int_{0}^{2 \pi}\left(\frac{\partial^{2} f}{\partial \theta \partial \phi}\right) g d \phi
$$


whence

$$
\begin{aligned}
\int_{\delta}^{\pi-\delta} d \theta \int_{0}^{2 \pi} \frac{\partial f}{\partial \theta} \frac{\partial g}{\partial \phi} d \phi & =-\int_{0}^{2 \pi} d \phi \int_{\delta}^{\pi-\delta}\left(\frac{\partial^{2} f}{\partial \theta \partial \phi}\right) g d \theta \\
& =-\int_{0}^{2 \pi}\left\{\left.\frac{\partial f}{\partial \phi} g\right|_{\delta} ^{\pi-\delta}-\int_{\delta}^{\pi-\delta} \frac{\partial f}{\partial \phi} \frac{\partial g}{\partial \theta} d \theta\right\} d \phi .
\end{aligned}
$$

Thus

$$
\begin{aligned}
\int_{\delta}^{\pi-\delta} d \theta \int_{0}^{2 \pi} \frac{1}{\sin \theta}\left(\frac{\partial f}{\partial \theta} \frac{\partial g}{\partial \phi}-\frac{\partial f}{\partial \phi} \frac{\partial g}{\partial \theta}\right) \sin \theta d \phi & \\
& =\left.\int_{0}^{2 \pi} g \frac{\partial f}{\partial \phi}\right|_{\theta=\delta} d \phi-\left.\int_{0}^{2 \pi} g \frac{\partial f}{\partial \phi}\right|_{\theta=\pi-\delta} d \phi .
\end{aligned}
$$

When $\delta \rightarrow 0$ the left side of the last equation tends to

$$
\int_{\Omega} \hat{\mathbf{r}} \cdot \nabla_{t} f \times \nabla_{t} g d \Omega
$$

The right side may be shown to tend to zero as follows. Let $g_{N}$ be the value of $g$ at the north pole $N$. Then, since $g$ is continuous at $N$,

$$
\left|g-g_{N}\right|<\epsilon
$$

for all $\delta<\delta_{0}$. Thus, if $\delta<\delta_{0}$,

$$
\left.\left|\int_{0}^{2 \pi} g \frac{\partial f}{\partial \phi}\right|_{\theta=\delta} d \phi|=| \int_{0}^{2 \pi}\left(g-g_{N}\right) \frac{\partial f}{\partial \phi}\right|_{\theta=\delta} d \phi \mid \leqq 2 \pi M \epsilon,
$$

where $M$ is a bound for $\partial f / \partial \phi$. Since $\epsilon>0$ is arbitrary this proves that

$$
\left.\lim _{\delta \rightarrow 0} \int_{0}^{2 \pi} g \frac{\partial f}{\partial \phi}\right|_{\theta=\delta} d \phi=0 .
$$

The second integral is shown to tend to zero in the same way.

Lemma 5.1 shows that

$$
\int_{\Omega} \mathbf{E}_{n} \cdot \overline{\mathbf{H}}_{m} d \Omega=0
$$

for all $n$ and $m$, completing the proof that the $\mathbf{E}_{n}$ and $\mathbf{H}_{n}$ are orthogonal. The uniqueness of a uniformly convergent expansion (5.2) follows immediately.

The multipole expansion for $\mathbf{A}$ will be obtained by expressing $\mathbf{A}$ in terms of its Debye potentials $u$ and $v$, expanding $u$ and $v$ in series of spherical harmonics, and showing that these series can be differentiated termwise. This procedure is justified by the following expansion theorem for scalar radiation functions.

Theorem 5.1. Let $u$ be a scalar radiation function for a domain $r>c$. Then $u$ has an expansion

$$
u(r)=\sum_{n=0}^{\infty} h_{n}(k r) S_{n}(\hat{\mathrm{x}})
$$


which converges in $r>c$ and may be differentiated termwise with respect to $r$ and $\hat{\mathbf{r}}$ any number of times. Moreover, the convergence of the original series and all its derivatives is uniform in any region $r \geqq c+\epsilon>c$.

The proof of Theorem 5.1 is based on several lemmas. The first is

Lemma 5.2. Let $u$ be a scalar radiation function for a domain $r>c$, and let $\alpha>c$. Then

$$
u(\mathbf{r})=\frac{\alpha^{2}}{4 \pi} \int_{\Omega}\left\{u\left(\alpha \hat{\mathbf{r}}^{\prime}\right) \frac{\partial}{\partial \alpha}\left(\frac{e^{i k R}}{R}\right)-\frac{e^{i k R}}{R} \frac{\partial u\left(\alpha \hat{\mathbf{r}}^{\prime}\right)}{\partial \alpha}\right\} d \Omega^{\prime}
$$

where

$$
R=\sqrt{r^{2}+\alpha^{2}-2 r \alpha \hat{\mathbf{r}} \cdot \hat{\mathbf{r}}^{\prime}}
$$

in the domain $r>\alpha$.

A proof of this lemma under the additional hypothesis that

$$
u(r \hat{\mathbf{r}})=O\left(\frac{1}{r}\right), \quad r \rightarrow \infty,
$$

uniformly in $\hat{\mathbf{r}}$ is well known [1, pp. 25-26]. A proof based on the present hypotheses has been given by the author in a recent paper [27]. Our next lemma is

Lemma 5.3. Let $f$ and $g$ be functions that are defined and of class $C^{1}$ on $\Omega$ and let

$$
F(\mathbf{r})=\int_{\Omega}\left\{f\left(\hat{\mathbf{r}}^{\prime}\right) \frac{e^{i k R}}{R}+g\left(\hat{\mathbf{r}}^{\prime}\right) \frac{\partial}{\partial \alpha}\left(\frac{e^{i k R}}{R}\right)\right\} d \Omega^{\prime}
$$

where

$$
R=\sqrt{r^{2}+\alpha^{2}-2 r \alpha \hat{\mathbf{r}} \cdot \hat{\mathbf{r}}^{\prime}}
$$

and $\alpha>0$. Then $F(\mathbf{r})$ has an expansion

$$
F(\mathbf{r})=\sum_{n=0}^{\infty} h_{n}(k r) S_{n}(\hat{\mathbf{r}})
$$

having the convergence properties described in Theorem 5.1 with $c=\alpha$.

The lemma that follows is needed in the proof of Lemma 5.3.

Lemma 5.4. If

$$
R=\sqrt{r^{2}+\alpha^{2}-2 r \alpha \cos \theta}
$$

then

$$
\frac{e^{i k R}}{i k R}=\sum_{n=0}^{\infty}(2 n+1) j_{n}(k \alpha) h_{n}(k r) P_{n}(\cos \theta)
$$

where $P_{n}(x)$ is the Legendre polynomial of degree $n$. The series converges for $0 \leqq \alpha<r$ and may be differentiated termwise with respect to $r, \alpha$, and $\theta$ any number 
of times. Moreover, the resulting series all converge absolutely and uniformly with respect to $r, \alpha$, and $\theta$ in any region $0<\alpha_{1} \leqq \alpha / r \leqq \alpha_{2}<1$.

Proof. Watson has shown [24, pp. 362-366] that (5.6) holds for all complex values of $k, r, \alpha$, and $\theta$ satisfying

$$
\left|k \alpha e^{i \theta}\right|<|k r| \text { and }\left|k \alpha e^{-i \theta}\right|<|k r| .
$$

An inspection of WATson's proof shows that the convergence is uniform in a slightly smaller domain including the set where $\alpha_{1} \leqq \alpha / r \leqq \alpha_{2}$ and $\theta$ is real. It follows from a well known result of function theory that the series may be differentiated termwise any number of times and that the resulting series converge uniformly on compact subsets of the domain of convergence (see, for example, KnopP's Theory of Functions, Volume I, p. 74).

Proof of Lemma 5.3. By Lemma 5.4

$$
\begin{aligned}
\frac{e^{i k R}}{R} & =i k \sum_{n=0}^{\infty}(2 n+1) j_{n}(k \alpha) h_{n}(k r) P_{n}\left(\hat{\mathbf{r}} \cdot \hat{\mathbf{r}}^{\prime}\right), \\
\frac{\partial}{\partial \alpha}\left(\frac{e^{i k R}}{R}\right) & =i k^{2} \sum_{n=0}^{\infty}(2 n+1) j_{n}^{\prime}(k \alpha) h_{n}(k r) P_{n}\left(\hat{\mathbf{r}} \cdot \hat{\mathbf{r}}^{\prime}\right),
\end{aligned}
$$

and the series converge uniformly in $\hat{\mathbf{r}} \cdot \hat{\mathbf{r}}^{\prime}$ for the given $\alpha$ and any $r>\alpha$. Hence the series may be integrated term by term with respect to $\hat{\mathbf{r}}^{\prime}$. This implies that $F(\mathbf{r})$ has an expansion (5.5) where

$S_{n}(\hat{\mathbf{r}})=i k(2 n+1) j_{n}(k \alpha) \int_{\Omega} f\left(\hat{\mathbf{r}}^{\prime}\right) P_{n}\left(\hat{\mathbf{r}} \cdot \hat{\mathbf{r}}^{\prime}\right) d \Omega^{\prime}$

$$
+i k^{2}(2 n+1) j_{n}^{\prime}(k \alpha) \int_{\Omega} g\left(\hat{\mathbf{r}}^{\prime}\right) P_{n}\left(\hat{\mathbf{r}} \cdot \hat{\mathbf{r}}^{\prime}\right) d \Omega^{\rho}
$$

and is obviously a spherical harmonic of order $n$. To complete the proof of the lemma we remark that, since $f$ and $g$ are of class $C^{1}$ and $e^{i k R} / R$ is of class $C^{\infty}$ in $r$ and $\hat{\mathbf{r}}$, the integral defining $F(\mathbf{r})$ may be differentiated under the integral sign any number of times. The derivatives of the series in equations (5.7) converge uniformly to the derivatives of their sums, by Lemma 5.4. Multiplying these series by $f$ and $g$, respectively, and integrating over $\Omega$ provides expansions for the derivatives of $F(\mathbf{r})$ that are clearly the term by term derivatives of (5.5). The uniform convergence of these series for $r \geqq \alpha+\epsilon>\alpha$ follows from Lemma 5.4 and the fact that $f$ and $g$ are bounded on $\Omega$.

It is now easy to prove Theorem 5.1, with the help of Lemmas 5.2 and 5.3. For, if $\alpha>c$ then $u$ is represented by (5.4) in $r>\alpha$, by Lemma 5.2. The functions $u$ and $\partial u / \partial r$ are of class $C^{1}$ on $r=\alpha$, because $u$ is analytic in $r>c$. Hence, by Lemma 5.3, $u$ has an expansion (5.3) in $r>\alpha$. But then, since $\alpha$ is any arbitrary number greater than $c$, (5.3) must converge in $r>c$. Lemma 5.2 also implies that (5.3) may be differentiated termwise and that (5.3) and all the derived series converge uniformly in $r \geqq \alpha+\delta>\alpha$, where $\alpha>c$ and $\delta>0$ are 
arbitrary. Thus if $\epsilon>0$ we may take $\alpha=c+\frac{1}{2} \epsilon, \delta=\frac{1}{2} \epsilon$. Then the convergence is uniform in $r \geqq c+\epsilon>c$, which completes the proof of Theorem 5.1.

Theorem 5.1 is now used to prove that every vector radiation function has a multipole expansions. This result is contained in

Theorem 5.2. Let A be a vector radiation function for a domain $r>c$. Then $\mathbf{A}$ can be written in one and only one way as a sum

$$
\mathbf{A}=\mathbf{E}_{1}+\mathbf{E}_{2}+\cdots+\mathbf{H}_{1}+\mathbf{H}_{2}+\cdots
$$

of electromagnetic multipoles. The series converges in $r>c$ and may be differentiated termwise any number of times. Moreover, the convergence of the original series and all of its derivatives is uniform in any region $r \geqq c+\epsilon>c$.

Proof. We know, by Theorem 4.1 and Corollary 4.2, that

$$
\mathbf{A}=\nabla \times(\nabla \times u \mathbf{r})+k \nabla \times v \mathbf{r}
$$

in $r>c$, where $u$ and $v$ are scalar radiation functions for $r>c$. By Theorem 5.1,

$$
\begin{aligned}
& u=\sum_{n=1}^{\infty} h_{n}(k r) S_{n}(\hat{\mathbf{r}}), \\
& v=\sum_{n=1}^{\infty} h_{n}(k r) T_{n}(\hat{\mathbf{r}}),
\end{aligned}
$$

the series having the convergence properties listed there. Substituting these series into (5.9) and differentiating termwise gives the expansion (5.8). The convergence properties claimed for the expansion (5.8) follow from Theorem 5.1. The uniqueness of the expansion follows from the orthogonality of the multipoles, as was remarked at the beginning of the section, since (5.8) converges uniformly.

6. Isolated Singularities of Vector Wave Functions. Let $L$ be a second order differential operator operating on functions $u(P)$ that are defined in some regions of space. A function $u$ is regular at a point $P_{0}$ (or is regular for $L$ at $P_{0}$ ) if it is defined, is of class $C^{2}$, and satisfies

$$
L u=0
$$

in some neighborhood of $P_{0}$. A function $u$ has an isolated singularity at $P_{0}$ if it is defined and regular in a deleted neighborhood of $P_{0}$, i.e., $u$ is defined and regular at all points other than $P_{0}$ in some neighborhood of $P_{0}$. Isolated singularities of solutions of the three dimensional Laplace equation

$$
\nabla^{2} u=0
$$

have been studied by KellogG [14, pp. 268-270]. His results may be summarized as follows. If $u$ has an isolated singularity at $P_{0}$ then

$$
u=u^{\prime}+u^{\prime \prime}
$$


near $P_{0}$, where $u^{\prime}$ is regular at $P_{0}$ and

$$
u^{\prime \prime}(P)=\frac{c}{r}+\frac{S_{1}(\hat{\mathbf{r}})}{r^{2}}+\frac{S_{2}(\hat{\mathbf{r}})}{r^{3}}+\cdots
$$

Here $\mathbf{r}=r \hat{\mathbf{r}}$ is a position vector relative to an origin at $P_{0}$ and $S_{n}(\hat{\mathbf{r}})$ is a spherical harmonic of order $n$. The term

$$
\frac{S_{n}(\hat{\mathbf{r}})}{r^{n+1}}
$$

is called an electrostatic multipole of order $n$. KELLOGG also showed that if

$$
u(P)=0\left(r^{-\mu}\right), \quad r \rightarrow 0,
$$

then the expansion of $u^{\prime \prime}(P)$ has only a finite number of terms, the highest order multipole having order $n$ not greater than $\mu-1$. As special cases of this result it is seen that if $\mu<1$ then $u$ has a removable singularity at $P_{0}$, while if $\mu<2$ then

$$
u(P)=u^{\prime}(P)+\frac{c}{r}
$$

near $P_{0}$.

These results have an immediate generalization to solutions of the Helmholtz equation

$$
\nabla^{2} u+k^{2} u=0
$$

We need only replace $r^{-(n+1)}$ in the multipole expansion by the spherical Hankel function

$$
h_{n}(k r)=\sqrt{\frac{\pi}{2 k r}} H_{n+\frac{1}{2}}^{(1)}(k r)
$$

and KELLOGG's proofs go through as before.

In this section exact analogues of these theorems are derived for isolated singularities of vector wave functions, i.e., solutions of the vector Helmholtz equation

$$
\nabla \times(\nabla \times \mathbf{A})-k^{2} \mathbf{A}=0 .
$$

The first result to be proved is

Theorem 6.1. Let $\mathbf{A}$ be a vector wave function that has an isolated singularity at a point $P_{0}$. Then, in a neighborhood of $P_{0}, \mathbf{A}$ can be written as a sum

$$
\mathbf{A}=\mathbf{A}^{\prime}+\mathbf{A}^{\prime \prime}
$$

of two wave functions, where $\mathbf{A}^{\prime}$ is regular at $P_{0}$ and $\mathbf{A}^{\prime \prime}$ is a radiation function for the domain whose complement is $P_{0}$. 
Our proof of Theorem 6.1 makes use of the integral identity

$$
\begin{aligned}
\int_{V}\{\mathbf{A} \cdot \nabla \times(\nabla \times \mathbf{B})-\mathbf{B} \cdot \nabla & \times(\nabla \times \mathbf{A})\} d V \\
& =\int_{S}\{(\nabla \times \mathbf{B}) \times \mathbf{A}-(\nabla \times A) \times \mathbf{B}\} \cdot \hat{\mathbf{n}} d S .
\end{aligned}
$$

This result, which may be called the second vector Green's identity, can be proved by applying the divergence theorem to the field $\mathbf{A} \times(\nabla \times \mathbf{B})$. Sufficient conditions for its validity are that $\mathbf{A}$ and $\mathbf{B}$ be of class $C^{2}$ in the closure of $V$ and that the boundary $S$ of $V$ be regular in the sense of KeLLOGG [14, p. 100].

A second important tool that is used in the proof of Theorem 6.1 is the freespace tensor Green's function $\boldsymbol{\Gamma}\left(P, P^{\prime}\right)$ for the vector Helmholtz equation. $\boldsymbol{\Gamma}$ is defined by*

$$
\boldsymbol{\Gamma}\left(P, P^{\prime}\right)=\mathrm{I} G\left(P, P^{\prime}\right)+\frac{1}{k^{2}} \nabla \nabla G\left(P, P^{\prime}\right)
$$

where $I$ is the unit tensor and

$$
G\left(P, P^{\prime}\right)=\frac{e^{i k R}}{4 \pi R},
$$

$R$ being the distance from $P$ to $P^{\prime}$. Let $\mathbf{u}$ be a constant vector. Then the field

$$
\mathbf{E}(P)=\boldsymbol{\Gamma}\left(P, P^{\prime}\right) \cdot \mathbf{u}=G\left(P, P^{\prime}\right) \mathbf{u}+\frac{1}{k^{2}} \nabla \frac{\partial G\left(P, P^{\prime}\right)}{\partial u},
$$

where $\partial G / \partial u=\nabla G \cdot \mathbf{u}$ is the directional derivative of $G$ in the direction $\mathbf{u}$, is a vector radiation function for the domain $P \neq P^{\prime}$. To see this note that both $G$ and $\partial G / \partial u$ satisfy the scalar Helmholtz equation. Hence

$$
\nabla \cdot \mathbf{E}=\nabla G \cdot \mathbf{u}+\frac{1}{k^{2}} \nabla^{2} \frac{\partial G}{\partial u}=\frac{\partial G}{\partial u}-\frac{\partial G}{\partial u}=\mathbf{0}
$$

and, therefore,

$$
\nabla \times(\nabla \times \mathbf{E})=-\nabla^{2} \mathbf{E}=k^{2} G \mathfrak{u}+\nabla \frac{\partial G}{\partial u}=k^{2} \mathbf{E},
$$

showing that $\mathrm{E}$ is a wave function for $P \neq P^{\prime}$. Moreover, a simple vector calculation gives

$$
r\{\hat{\mathbf{r}} \times(\nabla \times \mathbf{E})+i k \mathbf{E}\}=O\left(\frac{1}{r}\right), \quad r \rightarrow \infty,
$$

which implies the Silver-Müller radiation condition.

\footnotetext{
tensors.

"The dyadic notation of GIBBs-WILson "Vector Analysis" is used in discussions involving
} 
Before starting the proof of Theorem 6.1 we shall state and prove two lemmas which reveal the nature of the singularity of $\boldsymbol{\Gamma}$ at $P=P^{\prime}$. These results are indispensable in the applications of the tensor Green's function to boundary value problems.*

Let $S(\alpha)$ represent the surface of the sphere with center at $P^{\prime}$ and radius $\alpha$, and let $\hat{\mathbf{n}}$ be an outwardly directed unit normal to $S(\alpha)$. Then our first result is

Lemma 6.1. If $\mathbf{A}$ is any vector field that is continuous in a neighborhood of $P^{\prime}$, then

$$
\lim _{\alpha \rightarrow 0} \int_{S(\alpha)} \mathbf{A} \cdot \hat{\mathbf{n}} \times\{\nabla \times \mathbf{E}\} d S=\frac{2}{3} A\left(P^{\prime}\right) \cdot \mathbf{u} .
$$

Proof. Introduce a rectangular coordinate system $(x, y, z)$ with origin at $P^{\prime}$ and for which $\mathbf{u}=\hat{\mathbf{z}}$. (There is no loss of generality in assuming $|\mathbf{u}|=1$.) Then, if $(r, \theta, \phi)$ is the corresponding system of spherical coordinates,

$$
\mathbf{E}(P)=\frac{e^{i k r}}{4 \pi r} \hat{\mathbf{z}}+\frac{1}{k^{2}} \nabla \frac{\partial}{\partial z}\left(\frac{e^{i k r}}{4 \pi r}\right) .
$$

Hence

$$
\nabla \times \mathbf{E}=\nabla \times\left(\frac{e^{i k r}}{4 \pi r} \hat{\mathbf{z}}\right)=\nabla \frac{e^{i k r}}{4 \pi r} \times \hat{\mathbf{z}}=\left(\frac{e^{i k r}}{4 \pi r}\right)^{\prime} \hat{\mathbf{r}} \times \hat{\mathbf{z}}=-\left(\frac{e^{i k r}}{4 \pi r}\right)^{\prime} \sin \theta \hat{\boldsymbol{\phi}},
$$

where the prime means differentiation with respect to $r$. Thus

$$
I(\alpha)=\int_{S(\alpha)} \mathbf{A} \cdot \hat{\mathbf{n}} \times\{\nabla \times \mathbf{E}\} d S=-\left(i k-\frac{1}{\alpha}\right) \frac{e^{i k \alpha}}{4 \pi \alpha} \alpha^{2} \int_{\Omega} \mathbf{A} \cdot \hat{\mathbf{r}} \times(\sin \theta \hat{\mathbf{\phi}}) d \Omega,
$$

where, as in $\S 3, \Omega$ is the unit sphere with center $P^{\prime}$ and $d \Omega$ is the element of area on it. Since $\hat{\mathbf{r}} \times \hat{\boldsymbol{\phi}}=-\hat{\boldsymbol{\theta}}$ this may be written

$$
I(\alpha)=-(1-i k \alpha) \frac{e^{i k \alpha}}{4 \pi} \int_{\Omega} \mathbf{A} \cdot \hat{\boldsymbol{\theta}} \sin \theta d \Omega=-(1-i k \alpha) \frac{e^{i k \alpha}}{4 \pi}\left\{I_{1}(\alpha)+I_{2}(\alpha)\right\}
$$

where

$$
I_{1}(\alpha)=\int_{\Omega}\left(\mathbf{A}-\mathbf{A}_{0}\right) \cdot \hat{\boldsymbol{\theta}} \sin \theta d \Omega
$$

and

$$
I_{2}(\alpha)=\int_{\Omega} \mathbf{A}_{0} \cdot \hat{\boldsymbol{\theta}} \sin \theta d \Omega
$$

with $\mathbf{A}_{0}=\mathbf{A}\left(P^{\prime}\right)$. Now

$$
\left|I_{1}(\alpha)\right| \leqq \int_{\Omega}\left|\left(\mathbf{A}-\mathbf{A}_{0}\right) \cdot \hat{\boldsymbol{\theta}}\right| d \Omega
$$

$$
\leqq \int_{\Omega}\left|\mathbf{A}-\mathbf{A}_{0}\right| d \Omega \leqq 4 \pi \underset{P \varepsilon S(\alpha)}{\operatorname{Max}}\left|\mathbf{A}(P)-\mathbf{A}_{0}\right|
$$

* The lemmas and their proofs are contained in the author's doctoral thesis, Harvard University, 1955. 
At the second step in this inequality Schwarz's inequality was used. Since A is continuous at $P^{\prime}$ it is obvious that the last quantity can be made arbitrarily small by choosing $\alpha$ small enough. The second integral may be written

$$
I_{2}=\mathbf{A}_{0} \cdot \int_{\Omega} \hat{\boldsymbol{\theta}} \sin \theta d \Omega
$$

and

$$
\hat{\boldsymbol{\theta}}=\cos \theta(\cos \phi \hat{\mathbf{x}}+\sin \phi \hat{\mathbf{y}})-\sin \theta \hat{\mathbf{z}} .
$$

Hence, since $d \Omega=\sin \theta d \theta d \phi$ and $\int_{0}^{2 \pi} \sin \phi d \phi=\int_{0}^{2 \pi} \cos \phi d \phi=0$,

$$
\int_{\Omega} \hat{\boldsymbol{\theta}} \sin \theta d \Omega=-\hat{\mathrm{z}} \int_{0}^{\pi} \sin ^{3} \theta d \theta \int_{0}^{2 \pi} d \phi=-2 \pi \cdot \frac{4}{3} \hat{\mathrm{z}}=-\frac{8 \pi}{3} \hat{\mathrm{z}} .
$$

Thus $I_{2}(\alpha)=-(8 \pi / 3) \mathbf{A}_{0} \cdot \hat{\mathbf{z}}$ which, since $I_{1} \rightarrow 0$, proves that

$$
\lim _{\alpha \rightarrow 0} I(\alpha)=\frac{2}{3} \mathbf{A}\left(P^{\prime}\right) \cdot \mathbf{u} \text {. }
$$

Lemma 6.2. If $B$ is any vector field that is of class $C^{1}$ near $P^{\prime}$ then

$$
\lim _{\alpha \rightarrow 0} \int_{S(\alpha)} \mathbf{E} \times \mathbf{B} \cdot \hat{\mathbf{n}} d S=\frac{1}{3 k^{2}} \nabla \times \mathbf{B}\left(P^{\prime}\right) \cdot \mathbf{u} .
$$

Proof. Introduce coordinate systems as in the proof of Lemma 6.1. Write

$$
I(\alpha)=\int_{S(\alpha)} \mathbf{E} \times \mathbf{B} \cdot \hat{\mathbf{n}} d S=I_{1}(\alpha)+\frac{1}{k^{2}} I_{2}(\alpha)
$$

where

$$
I_{1}(\alpha)=\int_{S(\alpha)} \frac{e^{i k r}}{4 \pi r} \hat{\mathbf{z}} \times \mathbf{B} \cdot \hat{\mathbf{n}} d S
$$

and

$$
I_{2}(\alpha)=\int_{S(\alpha)} \nabla \frac{\partial}{\partial z}\left(\frac{e^{i k r}}{4 \pi r}\right) \times \mathbf{B} \cdot \hat{\mathbf{n}} d S .
$$

Then $I_{1}(\alpha)$ tends to zero with $\alpha$, for

$$
I_{1}(\alpha)=\frac{\alpha e^{i k \alpha}}{4 \pi} \int_{\Omega} \mathbf{B} \cdot \hat{\mathbf{r}} \times \hat{\mathbf{z}} d \Omega=-\frac{\alpha e^{i k \alpha}}{4 \pi} \int_{\Omega} \mathbf{B} \cdot \hat{\boldsymbol{\phi}} \sin \theta d \Omega .
$$

Since B is of class $C^{1}$ near $P^{\prime}$ it tends uniformly (in $\theta, \phi$ ) to its value at $P^{\prime}$ when $\alpha$ tends to zero. Hence the integral over $\Omega$ tends to a finite limit and $I_{1}$ tends to zero.

Now consider $I_{2}(\alpha)$. Since

$$
\frac{\partial}{\partial z}\left(\frac{e^{i k r}}{4 \pi r}\right)=\left(\frac{e^{i k r}}{4 \pi r}\right)^{\prime} \frac{\partial r}{\partial z}=\left(\frac{e^{i k r}}{4 \pi r}\right)^{\prime} \cos \theta
$$


we have

$$
\begin{aligned}
\nabla \frac{\partial}{\partial z}\left(\frac{e^{i k r}}{4 \pi r}\right) & =\left(\frac{e^{i k r}}{4 \pi r}\right)^{\prime \prime} \cos \theta \hat{\mathbf{r}}+\left(\frac{e^{i k r}}{4 \pi r}\right)^{\prime} \nabla \cos \theta \\
& =\left(\frac{e^{i k r}}{4 \pi r}\right)^{\prime \prime} \cos \theta \hat{\mathbf{r}}-\frac{1}{r}\left(\frac{e^{i k r}}{4 \pi r}\right)^{\prime} \sin \theta \hat{\boldsymbol{\theta}}
\end{aligned}
$$

Thus

$$
\hat{\mathbf{r}} \times \nabla \frac{\partial}{\partial z}\left(\frac{e^{i k r}}{4 \pi r}\right)=-\frac{1}{r}\left(\frac{e^{i k r}}{4 \pi f}\right)^{\prime} \sin \theta \hat{\boldsymbol{\phi}}
$$

and

$$
\begin{aligned}
I_{2}(\alpha) & =\int_{S(\alpha)} \mathbf{B} \cdot \hat{\mathbf{r}} \times \nabla \frac{\partial}{\partial z}\left(\frac{e^{i k r}}{4 \pi r}\right) d S \\
& =-\frac{1}{\alpha}\left(i k-\frac{1}{\alpha}\right) \frac{e^{i k \alpha}}{4 \pi \alpha} \alpha^{2} \int_{\Omega} \mathbf{B} \cdot \hat{\boldsymbol{\phi}} \sin \theta d \Omega \\
& =\left(\frac{1}{\alpha}-i k\right) \frac{e^{i k \alpha}}{4 \pi} \int_{0}^{\pi} \sin \theta d \theta \int_{0}^{2 \pi} \mathrm{B}(\alpha, \theta, \phi) \cdot \hat{\boldsymbol{\phi}} \sin \theta d \phi
\end{aligned}
$$

Now

$$
\alpha \int_{0}^{2 \pi} \mathbf{B} \cdot \hat{\boldsymbol{\phi}} \sin \phi d \phi=\int_{C} \mathbf{B} \cdot \hat{\mathbf{t}} d s
$$

where $C$ is the circle in the plane $z=\alpha \cos \theta$ with center at $x=0, y=0$ and radius $\alpha \sin \theta$, while $\hat{\mathrm{t}}=\hat{\boldsymbol{\phi}}$ is the unit tangent vector to $C$ and $d s=\alpha \sin \theta d \phi$ is the element of arc. By Stokes' theorem, which is applicable since $B$ is of class $C^{1}$, the last integral equals

$$
\int_{D}(\nabla \times \mathbf{B}) \cdot \hat{\mathbf{z}} d S
$$

where $D$ is the disc bounded by $C$ and $\hat{z}$ is its normal. Thus

$$
I_{2}(\alpha)=\left(\frac{1}{\alpha}-i k\right) \frac{e^{i k \alpha}}{4 \pi \alpha} \int_{0}^{\pi} \sin \theta d \theta \int_{0}^{\alpha \sin \theta} \rho d \rho \int_{0}^{2 \pi} F\left(\rho, \phi^{\prime}\right) d \phi^{\prime}
$$

where $\left(\rho, \phi^{\prime}\right)$ are polar coordinates in the plane $z=\alpha \cos \theta$ and $F\left(\rho, \phi^{\prime}\right)=$ $(\nabla \times \mathbf{B}) \cdot \hat{\mathbf{z}}$. Now

$$
\int_{0}^{\alpha \sin \theta} \rho d \rho \int_{0}^{2 \pi} F\left(\rho, \phi^{\prime}\right) d \phi^{\prime}=\alpha^{2} \sin ^{2} \theta \int_{0}^{1} \psi d \psi \int_{0}^{2 \pi} F\left(\alpha \sin \theta \psi, \phi^{\prime}\right) d \phi^{\prime}
$$

whence

$$
I_{2}(\alpha)=(1-i k \alpha) \frac{e^{i k \alpha}}{4 \pi} \int_{0}^{\pi} \sin ^{3} \theta d \theta \int_{0}^{1} \psi d \psi \int_{0}^{2 \pi} F\left(\alpha \sin \theta \psi, \phi^{\prime}\right) d \phi^{\prime} .
$$


Further, because $\mathrm{B}$ is of class $C^{1}, F\left(\alpha \sin \theta \psi, \phi^{\prime}\right)$ tends uniformly in $\theta, \phi^{\prime}$ to $\nabla \times \mathrm{B}\left(P^{\prime}\right) \cdot \hat{\mathbf{z}}$ when $\alpha \rightarrow 0$. Thus

$\lim _{\alpha \rightarrow 0} I_{2}(\alpha)=\frac{1}{4 \pi} \nabla \times \mathbf{B}\left(P^{\prime}\right) \cdot \hat{\mathbf{z}} \int_{0}^{\pi} \sin ^{3} \theta d \theta \int_{0}^{1} \psi d \psi \int_{0}^{2 \pi} d \phi^{\prime}=\frac{1}{3} \nabla \times \mathbf{B}\left(P^{\prime}\right) \cdot \mathbf{u}$,

which proves Lemma 6.2.

It is interesting to compare Lemma 6.2 with the corresponding result for the scalar Helmholtz equation. This would say that if $u$ is bounded near $P^{\prime}$ then

$$
\lim _{\alpha \rightarrow 0} \int_{S(\alpha)} u G d S=0
$$

It follows from the circumstance that $G$ has a singularity of order one at $P=P^{\prime}$. The fact that the corresponding integral for the vector Helmholtz equation has a non-zero limit may be anticipated from Corollary 6.3 below which says that a vector wave function cannot have an isolated singularity of order less than 2 .

The following corollary, which is a direct consequence of Lemmas 6.1 and 6.2, is needed in our proof of Theorem 6.1.

Corollary 6.1. If $\mathbf{A}$ is a vector field that is of class $C^{2}$ near $P^{\prime}$ then

$$
\begin{aligned}
\lim _{\alpha \rightarrow 0} \int_{S(\alpha)}\{\mathbf{E} \times(\nabla \times \mathbf{A})-\mathbf{A} \times(\nabla & \times \mathbf{E})\} \cdot \hat{\mathbf{n}} d S \\
& =\left\{\frac{1}{3 k^{2}} \nabla \times\left(\nabla \times \mathbf{A}\left(P^{\prime}\right)\right)+\frac{2}{3} \mathbf{A}\left(P^{\prime}\right)\right\} \cdot \mathbf{u} .
\end{aligned}
$$

If, in addition, A satisfies the vector Helmholtz equation at $P^{\prime}$ then

$$
\lim _{\alpha \rightarrow 0} \int_{S(\alpha)}\{\mathbf{E} \times(\nabla \times \mathbf{A})-\mathbf{A} \times(\nabla \times \mathbf{E})\} \cdot \hat{\mathbf{n}} d S=\mathbf{A}\left(P^{\prime}\right) \cdot \mathbf{u} .
$$

We are now ready to prove Theorem 6.1 . To begin let $(r, \theta, \phi)$ be a system of spherical coordinates with origin at $P_{0}$ and let $S_{1}$ and $S_{2}$ be spheres with centers at $P_{0}$ and radii $r_{1}, r_{2}$ satisfying $0<r_{1}<r_{2}$. The radius $r_{2}$ is assumed to be small enough so that $S_{2}$ lies within the domain of definition of $\mathrm{A}$. Apply the vector Green's identity to $\mathbf{A}(P)$ and $\mathbf{E}(P)=\boldsymbol{\Gamma}\left(P, P^{\prime}\right) \cdot \mathbf{u} . P^{\prime}$ may be any point other than $P_{0}$ inside $S_{2}$, and we assume that $r_{1}$ is so small that $P^{\prime}$ lies outside $S_{1}$. Now apply the integral identity in the region $V$ between $S_{1}$ and $S_{2}$ but excluding a small sphere $S(\alpha)$ of radius $\alpha$ and center $P^{\prime}$. The volume integral is zero because both $\mathbf{E}$ and $\mathbf{A}$ satisfy the vector Helmholtz equation in $V$. Thus

$$
\int_{S_{\mathbf{1}}+S_{\mathbf{2}}+S(\alpha)}\{(\nabla \times \mathbf{E}) \times \mathbf{A}-(\nabla \times \mathbf{A}) \times \mathbf{E}\} \cdot \hat{\mathbf{n}} d S=0
$$

where $\hat{\mathbf{n}}$ points out of the sphere $S_{2}$ and into the spheres $S_{1}$ and $S(\alpha)$. If we make $\alpha \rightarrow 0$ then the integral over $S(\alpha)$ tends to $-\mathbf{A}\left(P^{\prime}\right) \cdot \mathbf{u}$, by Corollary 6.1 . Hence

$$
\mathbf{A}\left(P^{\prime}\right) \cdot \mathbf{u}=\int_{S_{\mathbf{2}}+\mathbf{S}_{\mathbf{2}}}\{(\nabla \times \mathbf{E}) \times \mathbf{A}-(\nabla \times \mathbf{A}) \times \mathbf{E}\} \cdot \hat{\mathbf{n}} d S
$$


This may also be written

$$
\mathbf{A}\left(P^{\prime}\right) \cdot \mathbf{u}=\mathbf{u} \cdot \int_{S_{1}+S_{2}}\{(\nabla \times \mathbf{\Gamma}) \times \mathbf{A}+\Gamma \times(\nabla \times \mathbf{A})\} \cdot \hat{\mathbf{n}} d S .
$$

Since $\mathbf{u}$ is an arbitrary vector this implies

$$
\mathbf{A}(P)=\int_{S_{1}+S_{2}}\left\{\left(\nabla \times \boldsymbol{\Gamma}_{P P^{\prime}}\right) \times \mathbf{A}_{P^{\prime}}+\boldsymbol{\Gamma}_{P P^{\prime}} \times\left(\nabla \times \mathbf{A}_{P^{\prime}}\right)\right\} \cdot \hat{\mathbf{n}}_{P^{\prime}} d S
$$

where $\boldsymbol{\Gamma}_{P P^{\prime}} \equiv \mathbf{\Gamma}\left(P, P^{\prime}\right)$ and $\mathbf{A}_{P^{\prime}} \equiv \mathbf{A}\left(P^{\prime}\right)$. Let us write

$$
\mathbf{A}=\mathbf{A}^{\prime}+\mathbf{A}^{\prime \prime}
$$

where $\mathbf{A}^{\prime}$ is the integral over $S_{2}$ and $\mathbf{A}^{\prime \prime}$ is the integral over $S_{1}$. This decomposition of $\mathbf{A}$ will be shown to have the properties described in Theorem 6.1.

First, let $(x, y, z)$ be a system of rectangular coordinates. Then

$$
\boldsymbol{\Gamma}=\boldsymbol{\Gamma}_{1} \hat{\mathbf{x}}+\boldsymbol{\Gamma}_{2} \hat{\mathbf{y}}+\boldsymbol{\Gamma}_{3} \hat{\mathbf{z}}
$$

where $\boldsymbol{\Gamma}_{1}, \boldsymbol{\Gamma}_{2}$, and $\boldsymbol{\Gamma}_{3}$ are vector radiation functions for the domain $P \neq P^{\prime}$. It follows that if $\mathbf{u}$ and $\boldsymbol{\nabla}$ are any constant vectors then

$$
\boldsymbol{\Gamma} \times \mathbf{u} \cdot \mathbf{v} \quad \text { and } \quad(\nabla \times \boldsymbol{\Gamma}) \times \mathbf{u} \cdot \mathbf{v}
$$

are vector radiation functions for $P \neq P^{\prime}$. Now consider

$$
\mathbf{A}^{\prime}(P)=\int_{S_{\mathbf{a}}}\left\{\left(\nabla \times \boldsymbol{\Gamma}_{P P^{\prime}}\right) \times \mathbf{A}_{P^{\prime}}+\boldsymbol{\Gamma}_{P P^{\prime}} \times\left(\nabla \times \mathbf{A}_{P^{\prime}}\right)\right\} \cdot \hat{\mathbf{n}}_{P^{\prime}} d S^{\prime} .
$$

The integrand is a vector wave function for all $P \neq P^{\prime}$. Moreover, all the functions occurring in the integral are analytic and the integral may, therefore, be differentiated under the integral sign any number of times. It follows that $\mathbf{A}^{\prime}(P)$ is a vector wave function for the interior of $S_{2}$ and, in particular, is regular at $P=P_{0}$.

A similar argument shows that

$$
\mathbf{A}^{\prime \prime}(P)=\int_{S_{1}}\left\{\left(\nabla \times \boldsymbol{\Gamma}_{P P^{\prime}}\right) \times \mathbf{A}_{P^{\prime}}+\Gamma_{P P^{\prime}} \times\left(\nabla \times \mathbf{A}_{P^{\prime}}\right)\right\} \cdot \hat{\mathbf{n}}_{P^{\prime}} d{S^{\prime}}^{\prime}
$$

is a vector wave function for the domain exterior to $S_{1}$. Since the radius $r_{1}>0$ of $S_{1}$ is arbitrary it follows that $\mathrm{A}^{\prime \prime}$ is regular for all $P \neq P_{0}$. Finally, the integrand in $A^{\prime \prime}$ may be shown to satisfy the Silver-Müller radiation condition uniformly in $P^{\prime}$. Hence $\mathbf{A}^{\prime \prime}$ satisfies the radiation condition and so is a vector radiation function for $P \neq P_{0}$.

An immediate corollary of Theorems 6.1 and 5.2 is

Corollary 6.2. If $\mathbf{A}$ is a vector wave function that has an isolated singularity at $P_{0}$ then

$$
\mathbf{A}=\mathbf{A}^{\prime}+\mathrm{E}_{1}+\mathrm{E}_{2}+\cdots+\mathrm{H}_{1}+\mathrm{H}_{2}+\cdots
$$


where $\mathbf{A}^{\prime}$ is regular at $P_{0}$ while $\mathbf{E}_{n}$ and $\mathbf{H}_{n}$ are multipoles for the point $P_{0}$. The series converges for $r>0$ and has the convergence properties described in Theorem 5.2.

$A$ vector field $\mathbf{A}$ has a singularity of finite order at a point $P_{0}$ if

$$
\mathbf{A}(P)=O\left(r^{-\mu}\right), \quad r \rightarrow 0,
$$

where $\mu \geqq 0$ and $r$ is the distance from $P$ to $P_{0}$. Our next result states that if a vector wave function has an isolated singularity of finite order at $P_{0}$ then there are only a finite number of multipoles in the series (6.1). The precise result is:

Theorem 6.2. If $\mathbf{A}$ is a vector wave function that has an isolated singularity of finite order at a point $P_{0}$,

$$
\mathbf{A}(P)=O\left(r^{-\mu}\right), \quad r \rightarrow 0,
$$

then

$$
\mathbf{A}=\mathbf{A}^{\prime}+\sum_{n=1}^{[\mu-2]} \mathbf{E}_{n}+\sum_{n=1}^{[\mu-1]} \mathbf{H}_{n}
$$

where $\mathbf{A}^{\prime}$ is regular at $P_{0}$.

The order of an isolated singularity of finite order may be defined to be the smallest number $\mu$ for which (6.2) holds. Theorem 6.2 shows that the order exists and is an integer when $\mathbf{A}$ is a vector wave function. Before turning to the proof of Theorem 6.2 we give two lemmas which are needed in the proof.

Lemma 6.3. If $P_{n}(x)$ is the Legendre polynomial of degree $n$ and $S_{m}(\hat{\mathbf{r}})$ is a spherical harmonic of order $m$ then

$$
\int_{\Omega} S_{m}\left(\hat{\mathbf{r}}^{\prime}\right) P_{n}\left(\hat{\mathbf{r}} \cdot \hat{\mathbf{r}}^{\prime}\right) d \Omega^{\prime}=\frac{4 \pi \delta_{n m}}{2 n+1} S_{n}(\hat{\mathbf{r}}) .
$$

Proof. This is proved is Kellogg [14, p. 252].

Lemma 6.4. Under the hypotheses of Lemma 6.3,

$$
\int_{\Omega} \nabla_{t} S_{m}\left(\hat{\mathbf{r}}^{\prime}\right) \cdot \nabla_{t} P_{n}\left(\hat{\mathbf{r}} \cdot \hat{\mathbf{r}}^{\prime}\right) d \Omega^{\prime}=\frac{4 \pi n(n+1) \delta_{n m}}{2 n+1} S_{n}(\hat{\mathbf{r}}) .
$$

Proof. By Green's theorem for $\Omega$, [3, p. 174],

$$
\int_{\Omega} \nabla_{t} u \cdot \nabla_{t} v d \Omega=-\int_{\Omega} u \nabla_{t}^{2} v d \Omega
$$

Hence Lemma 6.4 follows from Lemma 6.3 and the equation $\nabla_{t}^{2} S_{n}=$ $-n(n+1) S_{n}$.

We are now ready to prove Theorem 6.2 . Write $\mathbf{A}=\mathbf{A}^{\prime}+\mathbf{A}^{\prime \prime}$ where $\mathbf{A}^{\prime}$ is regular at $P_{0}$ and $\mathbf{A}^{\prime \prime}$ is a radiation function for $r>0$. Notice that

$$
\mathbf{A}^{\prime \prime}(P)=O\left(r^{-\mu}\right), \quad r \rightarrow 0,
$$


with the same exponent $\mu$ as in (6.2), since $\mathbf{A}^{\prime}$ is regular at $r=0$. Let $u$ and $v$ be the Debye potentials for $A^{\prime \prime}$. They are scalar radiation functions for $r>0$, by Corollary 5.2, and hence (Theorem 5.1) have expansions

$$
\begin{aligned}
& u=h_{1} S_{1}+h_{2} S_{2}+\cdots, \\
& v=h_{1} T_{1}+h_{2} T_{2}+\cdots,
\end{aligned}
$$

valid in $r>0$. It follows from Corollary 4.1 and Theorem 5.1 that

$$
\mathbf{r} \cdot \mathbf{A}^{\prime \prime}=\sum_{n=1}^{\infty} n(n+1) h_{n} S_{n}
$$

Multiply this equation by $P_{n}\left(\mathbf{r} \cdot \mathbf{r}^{\prime}\right)$ and integrate over $\Omega$. The series may be integrated termwise and Lemma 6.3 gives

$$
r^{\mu-1} \int_{\Omega} \mathbf{r} \cdot \mathbf{A}^{\prime \prime} P_{n}\left(\hat{\mathbf{r}} \cdot \hat{\mathbf{r}}^{\prime}\right) d \Omega^{\prime}=\frac{4 \pi n(n+1)}{2 n+1} r^{\mu-1} h_{n}(k r) S_{n}(\hat{\mathbf{r}}) .
$$

Now

$$
h_{n}(x) \sim-i \frac{(2 n) !}{2^{n} n !} \frac{1}{x^{n+1}}, \quad x \rightarrow 0 .
$$

Moreover, the left side of (6.3) is bounded near $r=0$. This is impossible unless $S_{n}(\hat{\mathbf{x}}) \equiv 0$ for $\mu-1-(n+1)<0$ or $n>\mu-2$. Otherwise, if $S_{n}\left(\hat{\mathbf{r}}_{0}\right) \neq 0$ for some direction $\hat{\mathbf{r}}_{0}$, the right side of (6.3) is unbounded when $\hat{\mathbf{r}}=\hat{\mathbf{r}}_{0}$ and $r \rightarrow 0$ with $n>\mu-2$. Thus the terms of the expansions for $u$ can be different from zero only if $n \leqq \mu-2$.

The representation of $\mathbf{A}^{\prime \prime}$ by Debye potentials may now be written

$$
\mathbf{A}^{\prime \prime}=\sum_{n=1}^{[\mu-2]}\left\{k^{2} h_{n} S_{n} \hat{\mathbf{r}}+\nabla \frac{\partial\left(r h_{n} S_{n}\right)}{\partial r}\right\}+k \nabla v \times \mathbf{r} .
$$

Hence, since $\mathbf{r} \times \nabla f=\hat{\mathbf{r}} \times \nabla_{t} f$,

$$
\begin{aligned}
\hat{\mathbf{r}} \times \mathbf{A}^{\prime \prime} & =\sum_{n=1}^{[\mu-2]} \frac{1}{r} \hat{\mathbf{r}} \times \nabla_{t} \frac{\partial\left(r h_{n} S_{n}\right)}{\partial r}-k \hat{\mathbf{r}} \times\left(\hat{\mathbf{r}} \times \nabla_{t} v\right) \\
& =\frac{1}{r} \frac{\partial}{\partial r}\left(r \sum_{n=1}^{[\mu-2]} h_{n} \hat{\mathbf{r}} \times \nabla_{t} S_{n}\right)+k \nabla_{t} v .
\end{aligned}
$$

The gradient of the expansion for $v$ may be taken termwise. Thus

$$
\hat{\mathbf{r}} \times \mathbf{A}^{\prime \prime}=\frac{1}{r} \frac{\partial}{\partial r}\left(r \sum_{n=1}^{[\mu-2]} h_{n} \hat{\mathbf{r}} \times \nabla_{t} S_{n}\right)+k \sum_{n=1}^{\infty} h_{n} \nabla_{t} T_{n}
$$

If we take the scalar product of this equation with $\nabla_{t} P_{m}\left(\hat{\mathbf{r}} \cdot \hat{\mathbf{r}}^{\prime}\right)$ and integrate over $\Omega$ we get

$$
\begin{aligned}
\int_{\Omega} \mathbf{r}^{\prime} & \times \mathbf{A}^{\prime \prime} \cdot \nabla_{t} P_{m}\left(\hat{\mathbf{r}} \cdot \hat{\mathbf{r}}^{\prime}\right) d \Omega^{\prime} \\
& =\frac{1}{r} \frac{\partial}{\partial r}\left(r \sum_{n=1}^{[\mu-2]} h_{n} \int_{\Omega} \mathbf{r} \cdot \nabla_{t} S_{n} \times \nabla_{t} P_{m} d \Omega^{\prime}\right)+k \sum_{n=1}^{\infty} h_{n} \int_{\Omega} \nabla_{t} T_{n} \cdot \nabla_{t} P_{m} d \Omega^{\prime} .
\end{aligned}
$$


The integrals in the first sum all vanish, by Lemma 5.1 , and hence by Lemma 6.4

$$
r^{\mu} \int_{\Omega} \hat{\mathbf{r}}^{\prime} \times \mathbf{A}^{\prime \prime} \cdot \nabla_{t} P_{n}\left(\hat{\mathbf{r}} \cdot \hat{\mathbf{r}}^{\prime}\right) d \Omega^{\prime}=k \frac{4 \pi n(n+1)}{2 n+1} r^{\mu} h_{n}(k r) T_{n}(\hat{\mathbf{r}}) .
$$

If the argument following equation (6.3) is applied to (6.4) it is found that $T_{n}=0$ for $\mu-(n+1)<0$ or $n>\mu-1$. Thus the only non-zero terms in the expansion for $v$ occur for $n \leqq \mu-1$. This proves Theorem 6.2.

Two particular cases of Theorem 6.2 that are of special interest are stated as corollaries.

Corollary 6.3. If $\mathbf{A}(P)=O\left(r^{-\mu}\right)$ with $\mu<2$ then the singularity at $P_{0}$ is removable. In other words, there is no isolated singularity with order less than 2.

Proof. $\mu<2$ implies $\mu-1 \leqq 0$ and $\mu-2 \leqq 0$. Since the multipoles of order zero are identically zero this implies $\mathbf{A}^{\prime \prime}=0$. Hence $\mathbf{A}=\mathbf{A}^{\prime}$ is regular at $P_{\mathbf{0}}$.

Corollary 6.4. If $\mu<3$ then

$$
\mathbf{A}=\mathbf{A}^{\prime}+\nabla \times \frac{e^{i k r}}{r} \mathbf{u}
$$

where $\mathbf{u}$ is a constant vector. In other words, the only isolated singularity of order less than 3 is that of a dipole of magnetic type and has order 2.

Proof. $\mu<3$ implies $\mu-1 \leqq 1, \mu-2 \leqq 0$. Hence by Theorem 6.2 , the Debye potentials for $\mathbf{A}^{\prime \prime}$ are

$$
u=0, \quad v=h_{1} T_{1}
$$

where $T_{1}$ is a surface spherical harmonic of order 1 . The most general solid harmonic of order 1 may be written [14, p. 140] in the form

$$
\alpha x+\beta y+\gamma z,
$$

where $(x, y, z)$ are rectangular coordinates and $\alpha, \beta, \gamma$ are constants. If $\mathbf{u}=$ $\alpha \hat{\mathbf{x}}+\beta \hat{\mathbf{y}}+\gamma \hat{\mathbf{z}}$ then (6.5) may be written as $\mathbf{r} \cdot \boldsymbol{u}$. Hence $T_{1}$ may be written

$$
T_{1}=\hat{\mathbf{r}} \cdot \mathbf{u} \text {. }
$$

The representation of $\mathbf{A}^{\prime \prime}$ in terms of $u$ and $v$ becomes

$$
\mathbf{A}^{\prime \prime}=k \nabla \times v \mathbf{r}=k \nabla \times\left\{h_{1}(k r)(\hat{\mathbf{r}} \cdot \mathbf{u}) \mathbf{r}\right\} .
$$

Since $\nabla \times \mathbf{r}=0$ this may be written

$$
\mathbf{A}^{\prime \prime}=k \nabla\left(\hat{\mathbf{r}} \cdot \mathbf{u} h_{1}(k r)\right) \times \mathbf{r} .
$$

But

$$
\nabla\left(\hat{\mathbf{r}} \cdot \mathbf{u} h_{1}(k r)\right)=\frac{d}{d r}\left(\frac{h_{1}(k r)}{r}\right)(\mathbf{r} \cdot \mathbf{u}) \hat{\mathbf{r}}+\frac{h_{1}(k r)}{r} \mathbf{u}
$$


whence

$$
\begin{aligned}
\mathbf{A}^{\prime \prime} & =k \frac{h_{1}(k r)}{r} \mathbf{u} \times \mathbf{r}=-k h_{1}(k r)(\hat{\mathbf{r}} \times \mathbf{u})=h_{0}^{\prime}(k r)(\hat{\mathbf{r}} \times \mathbf{u}) \\
& =\frac{1}{k} \nabla h_{0}(k r) \times \mathbf{u}=\frac{1}{k^{2}} \nabla \times\left(\frac{e^{i k r}}{r} \mathbf{u}\right),
\end{aligned}
$$

since $h_{1}=h_{0}^{\prime}$ and $h_{0}=e^{i k r} / k r$. Thus

$$
\mathbf{A}^{\prime \prime}=\nabla \times\left(\frac{e^{i k r}}{r} \mathbf{u}\right)
$$

where $\mathbf{u}$ is a suitable constant vector.

\section{REFERENCES}

1. B. B. BAKer \& E. T. Copson, The Mathematical Theory of Huygen's Principle, 2nd Ed., Oxford (1950).

2. P. Bidal \& G. De Rham, Les formes différentielle harmoniques, Commentarii Mathematici Helvetici 19 (1956) pp. 1-49.

3. W. BLAschKe, Vorlesungen über Differentialgeometrie, J. Springer (1924).

4. C. W. Borchand, Untersuchungen über die Elasticität fester isotroper Körper unter Berücksichtigung der Wärme, Berliner Monatsberichte (1873), Collected Works pp. 247-288.

5. C. J. Bouwramp \& H. B. G. Casimir, On Multipole Expansions in the Theory of Electromagnetic Radiation, Physica 20 (1954) pp. 539-554.

6. H. Bremmer, Terrestrial Radio Waves, Elsevier Publishing Co., New York (1949).

7. A. Clemsch, Über die Reflexion an einer Kugelfläche, J. Reine Angew. Math. 61 (1863) pp. 195-262.

8. R. Courant \& D. Hilbert, Methods of Mathematical Physics, v. 1, Interscience Publishers Inc., New York (1953).

9. R. Courant \& D. Hilbert, Methoden der Mathematischen Physik, v. 2, J. Springer, Berlin (1937).

10. P. Debye, Der Lichtdruck auf Kugeln von beliebigem Material, Ann. d. Phys., 4th Series 30 (1909) pp. 57-136.

11. J. Hadamard, Lecons sur la propagation des ondes et les equations de l'hydrodynamique, Paris (1903).

12. D. HrLbert, Grundzuge einer allgemeinen Theorie der linearen Integralgleichungen, Leipzig and Berlin (1912).

13. E. W. Hobson, The Theory of Functions of a Real Variable, 2nd Ed., v. 2, Harren Press, Washington, D. C. (1950).

14. O. D. Kellogg, Foundations of Potential Theory, J. Springer, Berlin (1929).

15. H. Lamb, On the Oscillations of a Viscous Spheroid, Proc. London Math. Soc. 13 (1881) pp. 51-65.

16. H. LamB, On the Vibrations of an Elastic Sphere, Proc. London Math. Soc. 13 (1882) pp. 189-211.

17. H. LAMB, On the Motion of a Viscous Fluid contained in a Spherical Vessel, Proc. London Math. Soc. 16 (1884) p. 27.

18. W. Magnus \& F. Oberhettinger, Formulas and Theorems for the Special Functions of Mathematical Physics, Chelsea Publishing Co., New York (1949). 
19. J. Meixner, Strenge Theorie der Beugung elektromagnetischer Wellen an der vollkommen leitenden Kreisscheibe, Zeit. f. Naturforschung 3a (1948) pp. 506-518.

20. G. Mre, Beiträge zur Optik trüber Medien, speziell kolloidaler Metallösungen, Ann. $d$. Phys., 4th Series 25 (1908) pp. 377-445.

21. E. PICARD, Sur la détermination des integrales des certaines equations aux derivées partielles du second ordre par leurs valeurs le long d'un contour fermé, $J$. École Polytech. 60 (1890) pp. 89-105.

22. A. Sommerfeld, Elektromagnetische Schwingungen, in Die Differential- und Integralgleichungen der Mechanik und Physik, Part II, Vieweg, Braunschweig (1927) pp. 391-597.

23. J. J. Thomson, On the Scattering of Electric Waves by Metallic Spheres, Recent Researches in Electricity and Magnetism, Oxford (1893) pp. 437-451.

24. G. N. Watson, A Treatise on the Theory of Bessel Functions, 2nd Ed., Macmillan Co., New York (1944).

25. H. WeYL, Die natürlichen Randwertaufgaben in Aussenraum für Strahlungsfelder beliebiger Dimensionen und beliebigen Ranges, Math. Zeit. 56 (1952) pp. 105-119.

26. C. H. Wilcox, An Expansion Theorem for Electromagnetic Fields, Comm. Pure Appl. Math. 9 No. 2 (1956) pp. 115-134.

27. C. H. Wilcox, A Generalization of Theorems of Rellich and Atkinson, Proc. Amer. Math. Soc. 7 No. 2 (1956) pp. 271-276.

28. E. ZERMeLo, Hydrodynamische Untersuchungen uber die Wirbelbewegungen in einer Kugelfläche, Zeit. f. Math. u. Phys. 47 (1902) pp. 201-237.

Air Force Cambridge Research Center, Bedford, Massachusetts, and

California Institute of Technology,

Pasadena, California. 